\title{
Voxelwise Encoding Models Show That Cerebellar Language Representations Are Highly Conceptual
}

\author{
${ }^{(1)}$ Amanda LeBel, ${ }^{1}$ Shailee Jain, ${ }^{3}$ and Alexander G. Huth ${ }^{2,3}$ \\ ${ }^{1}$ Helen Wills Neuroscience Institute, University of California-Berkeley, Berkeley, California 94720 , ${ }^{2}$ Department of Neuroscience, University of \\ Texas-Austin, Austin, Texas 78712, and ${ }^{3}$ Department of Computer Science, University of Texas-Austin, Austin, Texas 78712
}

There is a growing body of research demonstrating that the cerebellum is involved in language understanding. Early theories assumed that the cerebellum is involved in low-level language processing. However, those theories are at odds with recent work demonstrating cerebellar activation during cognitive tasks. Using natural language stimuli and an encoding model framework, we performed an fMRI experiment on 3 men and 2 women, where subjects passively listened to $5 \mathrm{~h}$ of natural language stimuli, which allowed us to analyze language processing in the cerebellum with higher precision than previous work. We used these data to fit voxelwise encoding models with five different feature spaces that span the hierarchy of language processing from acoustic input to high-level conceptual processing. Examining the prediction performance of these models on separate BOLD data shows that cerebellar responses to language are almost entirely explained by high-level conceptual language features rather than low-level acoustic or phonemic features. Additionally, we found that the cerebellum has a higher proportion of voxels that represent social semantic categories, which include "social" and "people" words, and lower representations of all other semantic categories, including "mental," "concrete," and "place" words, than cortex. This suggests that the cerebellum is representing language at a conceptual level with a preference for social information.

Key words: cerebellum; computational; encoding; fMRI; language; semantic

Significance Statement

Recent work has demonstrated that, beyond its typical role in motor planning, the cerebellum is implicated in a wide variety of tasks, including language. However, little is known about the language representations in the cerebellum, or how those representations compare to cortex. Using voxelwise encoding models and natural language fMRI data, we demonstrate here that language representations are significantly different in the cerebellum compared with cortex. Cerebellum language representations are almost entirely semantic, and the cerebellum contains overrepresentation of social semantic information compared with cortex. These results suggest that the cerebellum is not involved in language processing per se, but cognitive processing more generally.

\section{Introduction}

The cerebellum is known to be involved in a diverse set of cognitive processes, including attention (Allen et al., 1997), working memory (Brissenden et al., 2018), object recognition (Liu et al., 1999), and language processing (Booth et al., 2007; Stoodley and

Received Jan. 18, 2021; revised Aug. 9, 2021; accepted Sep. 14, 2021.

Author contributions: A.L. and A.G.H. designed research; A.L. performed research; A.L. and S.J. analyzed data; A.L. wrote the first draft of the paper; A.L. and A.G.H. edited the paper; A.L. wrote the paper; S.J. contributed unpublished reagents/analytic tools.

This work was supported by the Whitehall Foundation, Alfred P. Sloan Foundation, Burroughs-Wellcome Fund, and the Texas Advanced Computing Center.

The authors declare no competing financial interests.

Correspondence should be addressed to Alexander G. Huth at huth@cs.utexas.edu.

https://doi.org/10.1523/JNEUROSCI.0118-21.2021

Copyright $\odot 2021$ LeBel et al.

This is an open-access article distributed under the terms of the Creative Commons Attribution 4.0 International license, which permits unrestricted use, distribution and reproduction in any medium provided that the original work is properly attributed.
Schmahmann, 2009). Evidence for the cognitive function of the cerebellum in healthy subjects has come largely from neuroimaging studies, which have found that certain cognitive tasks elicit consistently localized BOLD responses across cerebellum (King et al., 2019) and that resting-state BOLD fluctuations in cerebellum align to known resting-state networks in cortex (Buckner et al., 2011; Marek et al., 2018). However, little is known about what role the cerebellum plays in cognitive processes, or how representations in the cerebellum might differ from those found in cortex.

Language understanding is a highly complex cognitive process, which makes it a rich area of research to study cognitive processing. Hierarchically organized networks for language processing are widely distributed across much of cortex (Binder et al., 1997; Dronkers et al., 2004; Hickok and Poeppel, 2007; Poeppel et al., 2012; de Heer et al., 2017). These networks include some putative "language specific" areas in temporal and inferior frontal cortex (Fedorenko et al., 2011), as well as non-language- 


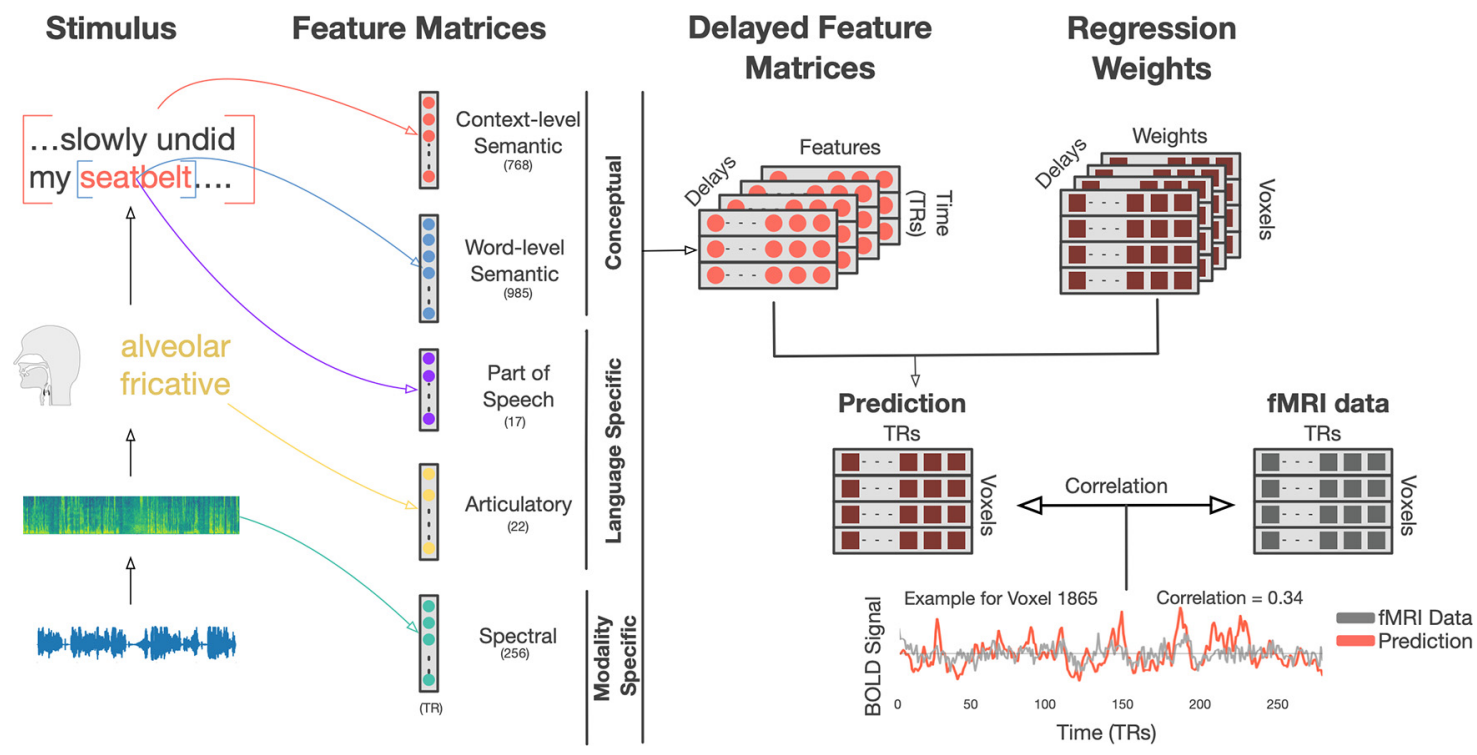

Figure 1. Voxelwise encoding model construction. To localize different stages of language processing across the cerebellum, we used five feature spaces to predict voxelwise BOLD responses in each subject: spectral, articulatory, part-of-speech, word-level semantic, and context-level semantic. Each 10-15 min stimulus story was transcribed and temporally aligned to the audio recording at the word and phoneme level. Features were then extracted for each of the five feature spaces. The features for the spectral model are 256 bands of a mel-frequency spectrogram. The features for the articulatory model are a 22 length $\mathrm{n}$-hot vector. The features for the part-of-speech model are a 1-hot 17 length vector. The features for the word-level semantic model are a 985-dimensional vector based on statistical word c0-occurrence. The features for the context-level semantic model are a 768 dimensional vector based on GPT (Radford et al., 2018), a neural network language model that incorporates context (preceding words) into the representation of the current word. Features were extracted for each time point, word, or phoneme, and concatenated into a feature matrix. The feature matrix was then resampled to the rate of the BOLD signal $(0.5 \mathrm{~Hz})$ and delayed to form an finite impulse response model that accounts for hemodynamics. Then regularized linear regression was used to fit weights that predict each voxel's BOLD signal from the stimulus matrix. Finally, models were used to predict responses on a held-out test dataset that was not used for model fitting. Model performance was assessed as the linear correlation between held-out BOLD data and model predictions for each voxel.

specific conceptual areas in temporal, parietal, and prefrontal cortex (Fedorenko et al., 2013). However, it is unclear whether these networks are also reflected in the cerebellum. Clinical evidence for a cerebellar role in language processing is found in work on cerebellar cognitive affective syndrome (CCAS), which shows that patients with acquired cerebellar damage experience language degradation, which can include agrammatism, dysprosody, and anomia (Schmahmann and Sherman, 1998). However, the subtlety and variability of these effects have made it difficult to form a complete picture. Early work into language deficits from cerebellar lesions has often conflicted with cases suggesting a degradation in grammar while preserving semantic content (Silveri et al., 1994; Justus, 2004; Frank et al., 2008) and other work suggesting a more uniform degradation in language processing that includes semantic content (Fiez et al., 1992; Silveri and Misciagna, 2000; Cook et al., 2004). However, it is unclear whether the standard aphasia tests used in these studies are sensitive enough to detect deficits from cerebellar damage (Cook et al., 2004; Murdoch, 2010). Our goal is to determine how language perception is localized in the cerebellum, what aspects of language are represented in the cerebellum, and how this compares to language processing systems in cortex.

Here we modeled cortical and cerebellar representations of natural speech using three different categories of features that span the putative language processing hierarchy (Hickok and Poeppel, 2007; de Heer et al., 2017): modality-specific, languagespecific, and conceptual. Modality-specific features capture information specific to how people perceive the language stimulus. In this study, subjects listened to audio recordings of naturally spoken narrative stories, so we used a feature space that captures frequency information in sound (Cheung et al., 2016). This feature space is known to be represented in auditory cortex (de Heer et al., 2017). Building on modality-specific features, language-specific features capture information that only exists in language, such as phoneme articulations and syntax. These feature spaces are known to be represented in superior temporal gyrus (STG) (Fedorenko et al., 2011; de Heer et al., 2017) and inferior frontal cortex (de Heer et al., 2017). Finally, conceptual features capture information about the meaning conveyed by language, which is known to be represented across broad regions of cortex, overlapping with other cognitive tasks (Fedorenko et al., 2013; de Heer et al., 2017). Previous work used similar methods to demonstrate that there is a hierarchy across these feature categories in cortex, where modality-specific information feeds into language-specific and then conceptual representations (de Heer et al., 2017). Here we investigated whether this hierarchy is replicated in the cerebellum or whether the cerebellum is specifically involved in only some aspects of language processing. For ease of language, "cortex" here refers exclusively to the cerebral cortex and "cerebellum" refers to the whole cerebellum, as cerebellar white matter was not excluded from analysis.

To determine which aspects of language the cerebellum is involved in processing or representing, we conducted a fMRI experiment where subjects passively listened to 27 natural, narrative stories $(5.4 \mathrm{~h})$ about a diverse set of topics. We then used voxelwise encoding models (Fig. 1) to determine how well each set of speech-related features could predict each voxel in each subject. The stimuli were first transformed into five different feature spaces: spectral, articulatory, part-of-speech, word-level semantic, and context-level (multiword) semantic. We used ridge regression to fit voxelwise encoding models with each feature space, and then tested how well these encoding models could predict responses to a new story that was not used for model fitting. Finally, we used variance partitioning to measure how much variance in cerebellar and cortical BOLD responses is uniquely explained by each of the five feature spaces. We found substantial evidence that the 
cerebellum represents language at a high conceptual and semantic level, and no strong evidence that the cerebellum represents any language-specific or modality-specific information.

In addition, we used the word-level semantic encoding models to determine whether the cerebellum represents different semantic categories than cortex. This analysis showed that all semantic categories are represented in both the cerebellum and cortex, but that the cerebellum has an overrepresentation of social semantic categories and an underrepresentation of mental, concrete, and place-related semantic categories compared with cortex.

\section{Materials and Methods \\ Participants}

Data were collected from 3 male subjects and 2 female subjects: UT-S-01 (female, age = $24 \mathrm{yr}$ ), UT-S-02 (male, age $34 \mathrm{yr}$ ), UT-S-06 (female, age 23 yr), UT-S-07 (male, age $=25 \mathrm{yr}$ ), UT-S-08 (male, age $=24 \mathrm{yr}$ ). Three of the subjects were authors (UT-S-01: S.J.; UT-S-02: A.G.H.; and UT-S-06: A.L.). All subjects were healthy and had normal hearing. The experimental protocol was approved by the Institutional Review Board at the University of Texas at Austin. Written informed consent was obtained from all subjects.

\section{fMRI collection}

MRI data were collected on a 3T Siemens Skyra scanner at the UT Austin Biomedical Imaging Center using a 64-channel Siemens volume coil. Functional scans were collected using gradient echo EPI with $\mathrm{TR}=2.00 \mathrm{~s}, \mathrm{TE}=30.8 \mathrm{~ms}$, flip angle $=71^{\circ}$, multiband factor (simultaneous multislice) $=2$, voxel size $=2.6 \mathrm{~mm} \times 2.6 \mathrm{~mm} \times 2.6 \mathrm{~mm}$ (slice thickness $=2.6 \mathrm{~mm}$ ), matrix size $=(84,84)$, and FOV $=220 \mathrm{~mm}$. FOV covered both the cortex and the cerebellum in their entirety for all subjects. Anatomical scans were collected using a T1-weighted multiecho MP-RAGE sequence on the same 3 T scanner with voxel size $=1 \mathrm{~mm} \times 1$ $\mathrm{mm} \times 1 \mathrm{~mm}$ following the Freesurfer morphometry protocol. Anatomical data for Subject UT-S-02 were collected on a 3T Siemens TIM Trio at the Berkeley Brain Imaging Center with a 32-channel Seimen's volume coil using the same sequence.

Known ROIs were localized separately in each subject. Three different tasks were used to define ROIs; these include a visual category localizer, an auditory cortex localizer, and a motor localizer.

For the visual category localizer, data were collected in six $4.5 \mathrm{~min}$ scans consisting of 16 blocks of 16 s each. During each block 20 images of either places, faces, bodies, household objects, or spatially scrambled objects were displayed. Subjects were asked to pay attention for the same image being presented twice in a row. The corresponding ROIs defined in cortex with this localizer were the fusiform face area (Kanwisher et al., 1997), occipital face area (Kanwisher et al., 1997), extrastriate body area (Downing et al., 2001), parahippocampal place area (Epstein and Kanwisher, 1998), and the occipital place area.

Motor localizer data were collected during two identical $10 \mathrm{~min}$ scans. The subject was cued to perform six different tasks in a random order in $20 \mathrm{~s}$ blocks. The cues were "hand," "foot," "mouth," "speak," "saccade," and "rest" presented as a word at the center of the screen, except for the saccade cue, which was presented as a random array of dots. For the hand cue, subjects were instructed to make small finger-drumming movements for the entirety of the time the cue was displayed. For the foot cue, the subjects were instructed to make small foot and toe movements. For the mouth cue, subjects were instructed to make small vocalizations that were nonsense syllables, such as balabalabala. For the speak cue, subjects were instructed to self-generate a narrative without vocalization. For the saccade cue, subjects were instructed to look around for the duration of the task.

Weight maps for the motor areas were used to define primary motor and somatosensory areas for the hands, feet, and mouth; supplemental motor areas for the hands and feet; secondary motor areas for the hands, feet, and mouth; and the ventral premotor hand area. The weight map for the saccade responses was used to define the frontal eye field and intraparietal sulcus visual areas. The weight map for the speech production was used to define Broca's area and the superior ventral premotor area speech area (Chang et al., 2011). In the cerebellum, weight maps for each subject were resliced in SUIT space (Diedrichsen, 2006), and then the resliced maps were averaged across subjects for each task. Motor areas for the hand, mouth, foot, and saccade tasks were defined in the posterior and anterior lobe.

Auditory cortex localizer data were collected in one $10 \mathrm{~min}$ scan. The subject listened to 10 repeats of 1 min auditory stimulus each containing $20 \mathrm{~s}$ of music (Arcade Fire), speech (Ira Glass, This American Life), and natural sound (a babbling brook). To determine whether a voxel was responsive to auditory stimulus, the repeatability of the voxel response across the 10 repeats was calculated using an $F$ statistic. This map was used to define the auditory cortex.

\section{fMRI preprocessing}

All functional data were motion-corrected using the FMRIB Linear Image Registration Tool (FLIRT) from FSL 5.0. (Woolrich et al., 2009) FLIRT was used to align all data to a template that was made from the average of all functional runs in the first story session for each subject. These automatic alignments were manually checked. Low-frequency voxel response drift was identified using a second-order Savitzky-Golay filter with a $120 \mathrm{~s}$ window and then subtracted from the signal. To avoid artifacts from onsets and poor detrending performance, responses were trimmed by removing $20 \mathrm{~s}$ (10 volumes) at the beginning and end of each scan. This removed the $10 \mathrm{~s}$ silent period as well as the first and last $10 \mathrm{~s}$ of each story. The mean response for each voxel was subtracted and the remaining response was scaled to have unit variance.

\section{Cortical surface reconstruction and visualization}

For cortical surfaces, meshes were generated from the T1-weighted anatomic scans using freesurfer (Dale et al., 1999). Before surface reconstruction, anatomic surface segmentations were hand-checked and corrected. Blender was used to remove the corpus callosum and make relaxation cuts for flattening. Functional images were aligned to the cortical surface using boundary-based registration implemented in FSL. These were checked for accuracy, and adjustments were made as necessary.

For the cerebellar cortical surfaces, the SUIT toolbox (Diedrichsen, 2006) was used to isolate the cerebellum from the rest of the brain using the T1-weighted anatomic image. The anatomical maps for the cerebellum were normalized into SUIT space using the SUIT registration algorithm. After encoding model fitting, cerebellar functional results were transformed into anatomic space and then resliced using SUIT. The SUIT flatmap and surface were added to the pycortex database for the purpose of surface visualization.

Model maps were created by projecting the values for each voxel onto the cortical surface using the "nearest" scheme in pycortex software (Gao et al., 2015). This projection finds the location of each pixel in the image in $3 \mathrm{D}$ space and assigns that pixel the associated value.

\section{Stimulus set}

The modeling training stimulus set consisted of 26 10-15 min stories taken from The Moth Radio Hour. In each story, a single speaker tells an autobiographical story without reading from a prepared speech. Each story was played during one scan with a buffer of $10 \mathrm{~s}$ on either side of the story start and stop. Data collection was broken up into 6 different days, the first session involving the anatomical scan and localizers, and each successive session consisting of 4 or 5 stories, plus one additional story used for model prediction. This additional story (which was not 1 of the 26 stories used for model training) was played in every session, and the responses to this story were averaged. Stories were played over Sensimetrics S14 in-ear piezoelectric headphones. The audio for each story was filtered to correct for frequency response and phase errors induced by the headphones using calibration data provided by sensimetrics and custom python code (https://github.com/alexhuth/sensimetrics_filter). All stimuli were played at $44.1 \mathrm{kHz}$ using the pygame library in Python.

Each story was manually transcribed by one listener. Certain sounds (e.g., laughter and breathing) were also marked to improve the accuracy of the automated alignment. The audio of each story was downsampled to $11 \mathrm{kHz}$, and the Penn Phonetics Lab Forced Aligner (P2FA) (Yuan, 2008) was used to automatically align the audio to the transcript. Praat (Boersma and Weenink, 2021) was then used to check and correct each aligned transcript manually. 
Feature spaces

Five feature spaces were used to cover the hierarchy of language processing. Each feature space was fit separately for each subject. The spectral feature space was a mel-band spectrogram with frequencies ranging from $\sim 0 \mathrm{~Hz}$ to $8 \mathrm{kHz}$ with 256 windows. The articulatory feature space was an $\mathrm{n}$-hot feature space where each phoneme is assigned a 1 for each articulation that is required to produce the sound and a 0 for every other articulation for a total of 22 features per phoneme. For the part-ofspeech feature space, a one-hot vector of 17 features was assigned to each word noting the part-of-speech for each word in each story. Partof-speech tagging was done using the flair package (Akbik et al., 2019). Flair is a language model that uses recurrent neural networks to tag speech into 17 categories (e.g., noun, verb, number, determiner, etc.). The wordlevel semantic space was a 985-dimensional feature space based on word co-occurrence (Huth et al., 2016). Each word in the stimulus set was assigned the vector associated with it in the original space. If the word in the story was not present in the original semantic space, it was assigned a vector of length 985 of zeros. The contextual semantic space was based on a finetuned GPT language model (Radford et al., 2018). GPT is a state-of-the-art language model that takes into account previous words while generating features for the current word. To assign features to each word, we extracted 768-dimensional feature vectors from layer 9 with a context length of 25 words. We chose layer 9 because it is a midlayer of GPT and it has been demonstrated that middle layers of recurrent language models are best able to predict brain activity (Jain and Huth, 2018; Toneva and Wehbe, 2019).

\section{Experimental design and statistical analysis}

Encoding model fitting. We used each of the 5 feature spaces to fit a linearized finite impulse response model to every cortical voxel in each subject. The cerebellar models and the cortical models were fit separately. The stimulus matrix for each story was downsampled using a 3-lobe Lanczos filter, then $z$-scored and concatenated together. To fit the linear model, the stimulus matrix has to account for variance in the hemodynamic response function across voxels. To do this, we concatenate four delayed copies of the stimulus (using delays of $1,2,3$, and 4 time points). This final stimulus matrix is then regressed with the BOLD data using ridge regression. We then test the model using a held-out dataset. This is done by taking the dot product of the weight matrix from the regression with the stimulus matrix from the held-out test set, resulting in a voxel $\times$ time point matrix. This resulting matrix is compared with the actual BOLD data for the held-out test set and the correlation calculated over time for each voxel to give a measure of model performance. The correlation was then noise-ceiling corrected for some analyses (noted in the text) (Schoppe et al., 2016). Total model performance metrics were computed using the mean $r^{2}$ across voxels. The $r^{2}$ was calculated as the signed $r^{2}(r \times|r|)$. Mean was used instead of summation to better account for the difference in number of voxels over the cerebellum compared with the cortex. To keep the scale of the weights consistent, a single value of the regularization coefficient was used for all voxels in both the cerebellum and cortex in all subjects. To find the best regularization coefficient, the regression procedure was bootstrapped 50 times in each subject; and a regularization performance curve was obtained for each subject by averaging the bootstrap sample correlations across the 50 samples, then across voxels, and finally across the 6 subjects, and the best overall value of the regularization parameter was selected. This was done separately for each feature space.

Individual model comparison. Encoding models with each of the feature spaces were fit in the cerebellum and cortex in each subject, and the regression weights were used to predict a held-out test set. Model performance for each voxel was estimated by taking the correlation of the predicted time series for each voxel with the actual data. Then to test whether the model performance was significantly $>0$, the time series for each voxel was randomly shuffled in blocks of 10 TRs and the correlation with the predicted time series was recalculated. This was done for 10,000 permutations to gain a null distribution of responses. This null distribution was used to calculate the $p$ value for each voxel and this was FDRcorrected to account for multiple comparisons. A threshold of $q(\mathrm{FDR})$ $<0.05$ was used to test for significantly well-predicted voxels. This was done individually in the cerebellum and cortex in each subject for each model. The correlations were also noise-ceiling corrected. Comparison was done across subjects by taking the average $r^{2}$ of all voxels in each subject in the cerebellum and cortex.

Noise ceiling correction. Noise ceiling correction was done using a modified normalized correlation coefficient $\left(C C_{\text {norm }}\right)$ (Schoppe et al., 2016). This was calculated by first calculating the product-moment correlation defined as follows:

$$
C C_{a b s}=\frac{\operatorname{Cov}(X, Y)}{\sqrt{\operatorname{Var}(X) \operatorname{Var}(Y)}}
$$

Where $X$ are the neural representations after repeat stimuli presentation and $Y$ are the model predictions. Then, to isolate model performance from prediction accuracy, this was normalized by the following:

$$
\begin{gathered}
C C_{\text {norm }}=\frac{C C_{a b s}}{C C_{\max }} \text { with } \\
C C_{\max }=\sqrt{\frac{2}{1+\sqrt{\frac{1}{C C_{\text {half }}^{2}}}}}
\end{gathered}
$$

The $C C_{\max }$ is the maximum correlation coefficient between the recorded BOLD signal and the best prediction that a perfect model could theoretically achieve. In addition to this standard $C C_{\text {norm }}$, we took the maximum with an added maximum flooring of 0.3 , which was experimentally determined to result in the most normally distributed correlations. Without the maximum flooring parameter, the estimated correlation after noise ceiling correction would go $>1$.

Variance partitioning. Because five-way variance partitioning has too many partitions to be interpretable, we used two versions of variance partitioning to test specific hypotheses. The first version looked at the unique variance explained by each model. This was done to test whether one feature space is uniquely better at predicting cerebellar or cortical voxels. The second version was a pairwise variance partitioning where each model was jointly fit with the contextual semantic space. This was done to test for the specific hypothesis that the contextual semantic model is better predicting the same areas as the low-level models in the cerebellum; that is, are there unique low-level language representations in the cerebellum or is the contextual semantic model better predicting the same areas as the low-level models? To do variance partitioning, joint models with the concatenated feature spaces are fit and then used to predict the held-out dataset. To be succinct, the variance explained by the five feature spaces will be written as Sets A-E.

Unique partition. The following nested models were fit:

$$
\begin{gathered}
A \cup B \cup C \cup D \cup E, A \cup B \cup C \cup D, A \cup B \cup C \cup E, A \cup B \cup D \cup E, \\
A \cup C \cup D \cup E \text {, and } B \cup C \cup D \cup E
\end{gathered}
$$

The variance uniquely explained by each feature space without any overlap from the other feature spaces, or relative complement (RC), was then calculated for each feature space as follows:

$$
\begin{aligned}
& A^{R C}=A \cup B \cup C \cup D \cup E-B \cup C \cup D \cup E, \\
& B^{R C}=A \cup B \cup C \cup D \cup E-A \cup C \cup D \cup E \\
& C^{R C}=A \cup B \cup C \cup D \cup E-A \cup B \cup D \cup E \\
& D^{R C}=A \cup B \cup C \cup D \cup E-A \cup B \cup C \cup E \\
& E^{R C}=A \cup B \cup C \cup D \cup E-A \cup B \cup C \cup D
\end{aligned}
$$

A Fisher-corrected permutation test with 10,000 permutations was done in each subject in both the cerebellum and cortex for each voxel for the unique partitions using the joint $A \cup B \cup C \cup D \cup E$ model. Multiple 
comparison correction was done using FDR with a threshold of $p<0.05$. Cerebellar data were resliced after the calculation of the unique partitions and the significance testing. The mean of the variance explained was calculated for each subject, in each partition, in the cerebellum and cortex.

To calculate whether each partition was significantly $>0$, we used a permutation test with 1000 permutations. To develop a null hypothesis of zero unique variance explained, we permuted blocks (block length of 10 TRs) of the model prediction for the BOLD activity for each mode. We then calculated the correlation of the permuted prediction with the actual held-out BOLD activity. Block permutation preserves autocorrelation statistics of the time series (Kunsch, 1989) and thus provides a sensible null hypothesis for these significance tests. We then recalculated the unique variance and nonunique partition, performed the bias correction as described below, and then took the mean unique variance explained for each partition. We repeated this process 1000 times to create a null distribution for each unique partition and the nonunique partition. This was done separately for cerebellum and cortex.

To determine whether the difference in partition sizes between cerebellum and cortex was significantly different from zero, we first concatenated the unique variance explained by the model for all significantly well-predicted voxels in both cerebellum and cortex into a single vector. For each of 10,000 permutations, we shuffled this vector and then resplit it into "cerebellar" and "cortical" groups, that is, shuffled the label (cortex vs cerebellum) assigned to each voxel. We then found the difference in the means between these two groups. Finally, we compared the actual mean difference value to the distribution of values obtained from permutations to calculate the $p$ value.

Pairwise variance partitioning. The following concatenated models were fit (where $\mathrm{A}$ is the contextual semantic feature space):

$$
A \cup B, A \cup C, A \cup D, A \cup E, A, B, C, D, E
$$

The variance explained by the intersections were calculated as follows:

$$
\begin{aligned}
& A \cap B=A+B-A \cup B \\
& A \cap C=A+C-A \cup C \\
& A \cap D=A+D-A \cup D \\
& A \cap E=A+E-A \cup E
\end{aligned}
$$

Then the unique contribution of each feature space in each pair can be calculated. This is the unique contribution without overlap from the other feature space noted as $R C / X$ where $X$ is the other paired feature space. These are calculated as follows:

$$
\begin{gathered}
A^{R C / B}=A \cup B-B \\
B^{R C}=A \cup B-A \\
A^{R C / C}=A \cup C-C \\
C^{R C}=A \cup C-A \\
A^{R C / D}=A \cup D-D \\
D^{R C}=A \cup D-A \\
A^{R C / E}=A \cup E-E \\
E^{R C}=A \cup E-A
\end{gathered}
$$

A Fisher-corrected permutation test with 10,000 permutations was done in each subject in both the cerebellum and cortex for each voxel for the unique partitions and intersections using the joint $A \cup B, A \cup C, A \cup D, A \cup E$ models. Multiple comparison correction was done using FDR with a threshold of $q(\mathrm{FDR})<0.05$. Cerebellar data were resliced after the calculation of the unique partitions and the significance testing.

Correction of variance partition estimates. Because empirical estimates of variance explaining contain sampling noise and the larger joint models are more prone to this noise, the set theoretical approach described above can result in theoretically impossible results. These theoretically impossible results can present as the group models explaining less variance than the individual models. This is because of the increase in the number of features but the amount of data being constant. To correct this problem, a post hoc correction was applied to the estimated variance explained by each model (de Heer et al., 2017). This correction moved the estimates to the nearest values that produced no nonsensical results. Mathematically, this involved estimating a bias term for the variance explained by each model in each voxel. We assumed that the estimated variance explained by some model $\left(r^{2}\right), X^{*}: \hat{X}=X^{*}+b_{x}$.

For the pairwise models, there are three bias parameters (one for each individual feature space and one for the combined model). For the unique variance-explained paradigm, there are six bias parameters (one for the five-way combined model, and one for each individual feature space in a leave one out paradigm). Further, because we know that the size of each variance partition must be at least equal to zero, the set theory equations that give the size of each partition can be used to define the inequality constraints on the bias terms. Assume that we want to find the smallest set of bias parameters that produce no nonsensical results, this allowed us to set up a constrained function minimization problem as follows:

$$
\min \left\{\|b\|^{2} \operatorname{subjecttoh}_{j}(b) \geq 0 \text { forj }=1 \ldots . x\right.
$$

Where $h$ indicates our inequality constraints and $x$ is the number of bias parameters.

This procedure was applied separately to the estimated values of the variance explained for each voxel. This procedure is adapted from the one used by de Heer et al. (2017).

Analysis of model weights. To assess similarity of semantic categories between cortex and cerebellum, the semantic space had to be broken into discrete categories instead of a smoothly continuous space. To do this the encoding model weights for the top $25 \%$ of voxels predicted by the word-level semantic model in each subject were concatenated together across subjects. This was done separately in cortex and cerebellum, and then those were also concatenated together. Then the model weights were normalized across voxels and principal components analysis was used to drop the number of dimensions from 985 to 86 , which we chose because it explained $80 \%$ of the variance. These data were then clustered using spherical $k$-means into 5 clusters. Cluster labels were determined subjectively based on the most similar words to the cluster centroid. To create an additional label set not biased by the authors, we asked 8 observers to provide five possible category labels based on the 10 words closest to the cluster centroid. We then averaged these responses using word2vec and have provided these labels in Extended Data Figure 6-3.

To choose the number of clusters, we calculated inertia, which is the within-cluster sum of squares criterion, of the clustering algorithm for a range of clusters between 1 and 20 clusters. From this, we calculated the point where the inertia changes from an exponential drop to a linear drop in inertia. This can also be defined as the point where the inertia is farthest from a linear line connecting the inertia at cluster 1 to the inertia at cluster 20. This point occurred at 5 clusters. (Extended Data Fig. 6-1 shows the inertia across all clusters tested.)

To test for significance in category differences between cerebellum and cortex, a permutation test was done by shuffling voxels between the cortex and the cerebellum for each subject. The difference in the ratio of each category in the cerebellum compared with the ratio of that category in the cortex was calculated for both the permutation set and the original data. The two-tailed $p$ value was calculated for each category as the ratio of the permutation difference greater than the absolute value of the original data difference plus the ratio of the permutation difference less than 
the negative absolute value of the original data. This was multiple comparison corrected using FDR with a threshold of $p<0.05$.

Data availability

The data used in this study is publicly available on OpenNeuro (LeBel et al., 2021). A corresponding dataset paper is being prepared to go along side the data.

\section{Results}

\section{Encoding model performance}

To determine which aspects of language might be processed in the cerebellum, we created five feature spaces that span the hierarchy of language processing from sound to context-level meaning, including a spectral feature space, an articulatory space, a part-of-speech space, a word-level semantic space, and a contextlevel semantic space that combines information across words. Previous work has demonstrated that these feature spaces can capture these different components of language and predict BOLD responses in cortex (Huth et al., 2016; de Heer et al., 2017; Jain and Huth, 2018). We fit separate encoding models with each feature space using $5.4 \mathrm{~h}$ of BOLD responses recorded while subjects listened to 26 different natural narrative stories taken from The Moth Radio Hour. Then, each model was used to predict responses to a different $10 \mathrm{~min}$ story, and model performance was quantified as the correlation between the predicted and actual BOLD responses $\left(r^{2}\right)$. Figure $2 A$ shows the prediction performance values for each feature space in one subject projected onto the SUIT cerebellar surface as well as prediction performance of each model in the cortex (similar maps for other subjects are in Extended Data Fig. 2-1).

The spectral model uses a 256-dimensional, modality-specific feature space representing a mel-frequency spectrogram. This feature space is highly predictive of the primary auditory cortex along the transverse temporal gyrus. It does not significantly predict any voxel in the cerebellum (one-sided permutation test, $q$ $($ FDR $)<0.05)$, but it does appear to have diffuse low prediction performance across lobules VIIA, VIIB, and VIIIA. Of note, this is similar to previous results that showed cerebellar response to auditory stimulus along the medial portion of these lobules (Snider and Stowell, 1944). However, there appears to be no clustering of spectrally selective voxels in the cerebellum, as is seen in the auditory cortex. To confirm that our result is not merely because of this feature space missing the representations that could capture low-level auditory processing in the cerebellum, we also fit the spectrotemporal model as described previously (Norman-Haignere and McDermott, 2018). This more advanced model does not explain more variance in the cerebellum than the spectral model (Extended Data Fig. 2-3). This suggests that the cerebellum has no homologous area to the primary auditory cortex.

The articulatory model uses a 22-dimensional binary, language-specific feature space, with each dimension representing 1 of the 22 articulations used in English (e.g., bilabial, back) (Levelt, 1993). In cortex, the articulatory space best predicts lateral, posterior temporal cortex along superior temporal gyrus. In the cerebellum, this feature space has diffuse prediction performance across lobules VIIIA and VIIIB, and significantly predicts a limited number of voxels in the medial posterior cerebellum (one-sided permutation test, $q(\mathrm{FDR})<0.05$ ). These areas are not traditionally considered motor speech areas (Callan et al., 2006; Manto et al., 2012); thus, this is unlikely to be because of covert rehearsal. This suggests that the cerebellum is not merely representing the articulations required to produce speech, and the lack of spatial clustering of well-predicted voxels further supports a lack of a homologous area to the auditory cortex.

The part-of-speech model uses a 17-dimensional binary, language-specific feature space, where each dimension represents 1 of 17 lexical classes (e.g., noun, verb, adjective). This feature space weakly but significantly predicts voxels covering a wide area of the cortex (one-sided permutation test, $q(\mathrm{FDR})<0.05$ ), including much of the frontal, temporal, and parietal lobes, with peak performance along the superior temporal lobe and near the intraparietal sulcus. In the cerebellum, this model significantly predicts voxels in many areas of the posterior lobe, with the highest model prediction performance in Crus I and II. This is a midlevel, language-specific feature space, and its performance suggests that the cerebellum is largely representing information at a higher level than sound or articulations.

The word-level semantic model uses a 985-dimensional conceptual feature space that is based on word co-occurrence statistics across a large corpus of written English (Huth et al., 2016; de Heer et al., 2017; Deniz et al., 2019). This feature space captures semantic information under the assumption that words that frequently occur in similar contexts carry similar meaning (Firth, 1957). The word-level semantic model predicts cortical voxels across regions in the frontal, parietal, and temporal lobes beyond core language-specific regions. In the cerebellum, this model significantly predicts voxels in Crus I and II and lobules VIIIA and VIIIB. This conceptual model predicts much more response variance in the cerebellum and cortex than do lower-level models.

The best model in both the cerebellum and cortex is the context-level semantic model. This model builds on the word-level conceptual model by combining information across words. It uses the hidden state of a neural language model as a feature space. Neural language models are artificial neural networks that learn to predict the next word in a sequence from past words. As a consequence, they learn a word's meaning in context, improving on the word-level model, which is context-invariant (Lin et al., 2019; Radford et al., 2019; Tenney et al., 2019). Here, we used GPT (Jain and Huth, 2018; Radford et al., 2018), which is a popular neural language model. The feature space is 768-dimensional, and the features are extracted from a middle layer of the language model that has previously been shown to be highly effective at predicting brain responses (Toneva and Wehbe, 2019). For each word, the past 25 words are used as context in the model. The context-level semantic model significantly predicts the largest number of voxels and most total variance across cortex, with peak prediction performance in frontal, parietal, and temporal cortex. In the cerebellum, this model yields very high prediction performance across most of the posterior cerebellum, including Crus I and II and lobules VIIIA and VIIIB.

To compare model performance between the cerebellum and cortex directly, we computed the average performance of each model in the cerebellum and cortex for each subject. Figure $2 B$ shows that there is a linear relationship between model performance in the cerebellum and cortex, suggesting that language might be represented similarly in these two structures. To account for the possibility that BOLD signal-to-noise varies systematically between cortex and cerebellum, we also adjusted the estimated correlation for each voxel using a standard technique (Schoppe et al., 2016). Figure 2C shows these results when accounting for the difference in signal-to-noise variance between cortex and cerebellum. Here, the pattern of results is largely the same, but prediction performance in the cerebellum is more similar to that of cortex. In both cases, however, cerebellar voxels that are well predicted by each feature space are highly 
A

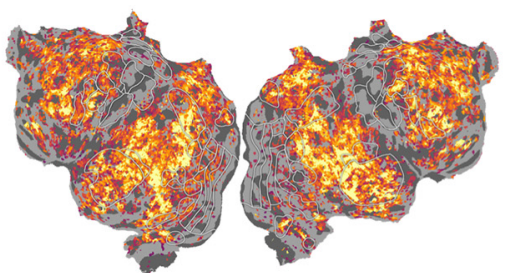

Context-level

Semantic

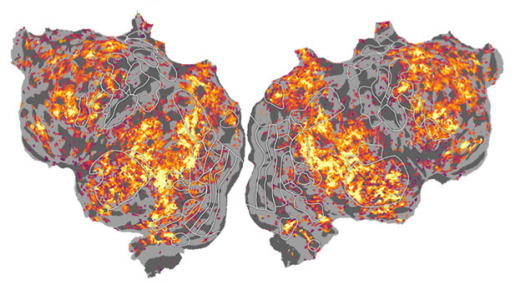

Word-level

Semantic
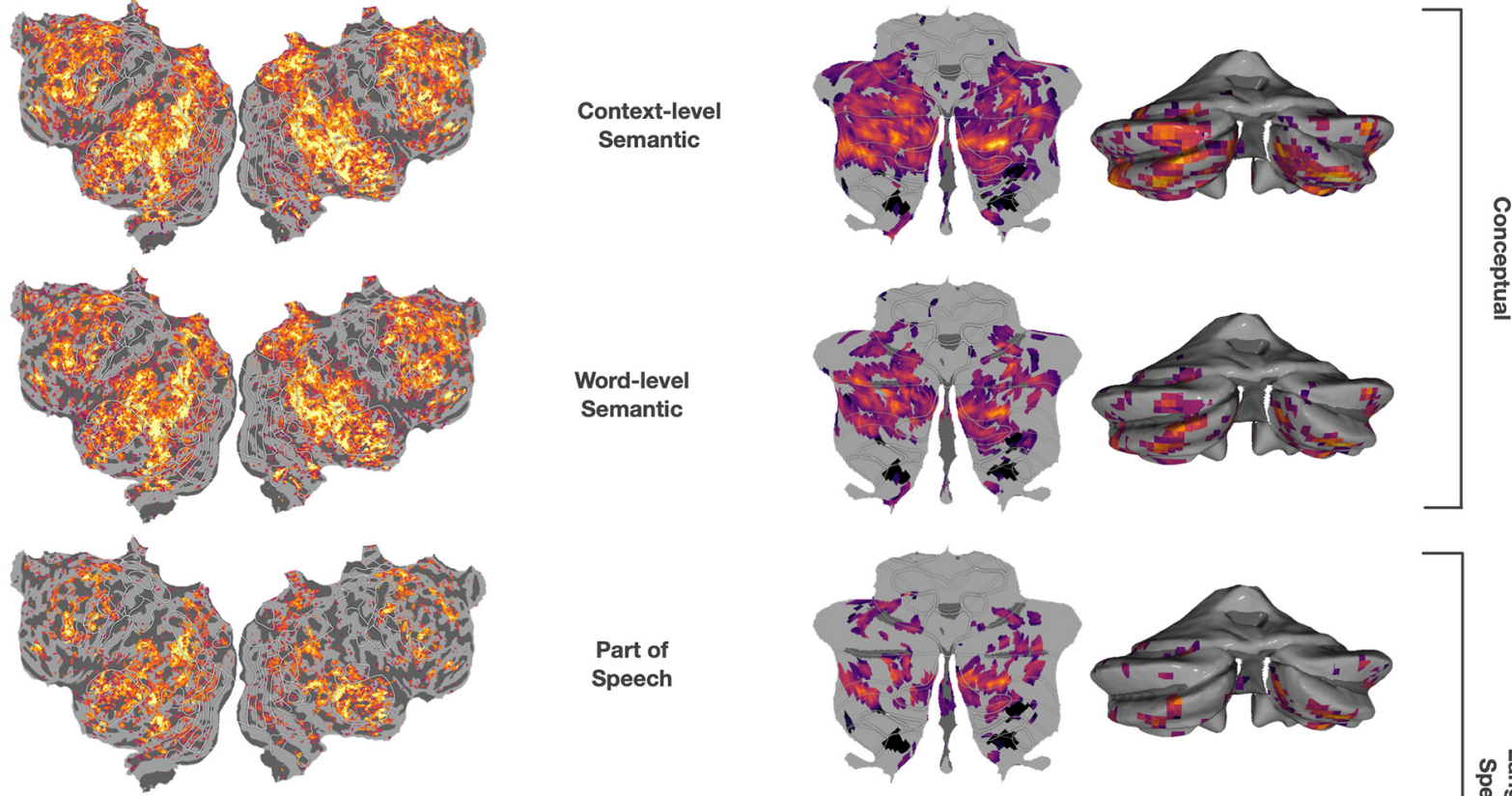

Part of
Speech
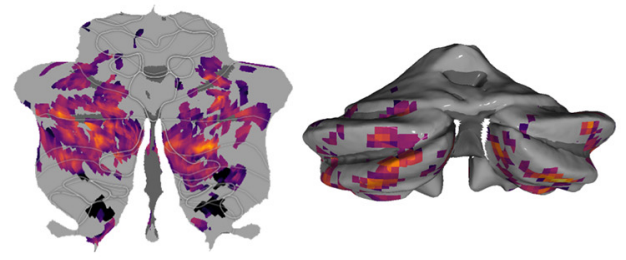

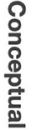

Articulatory
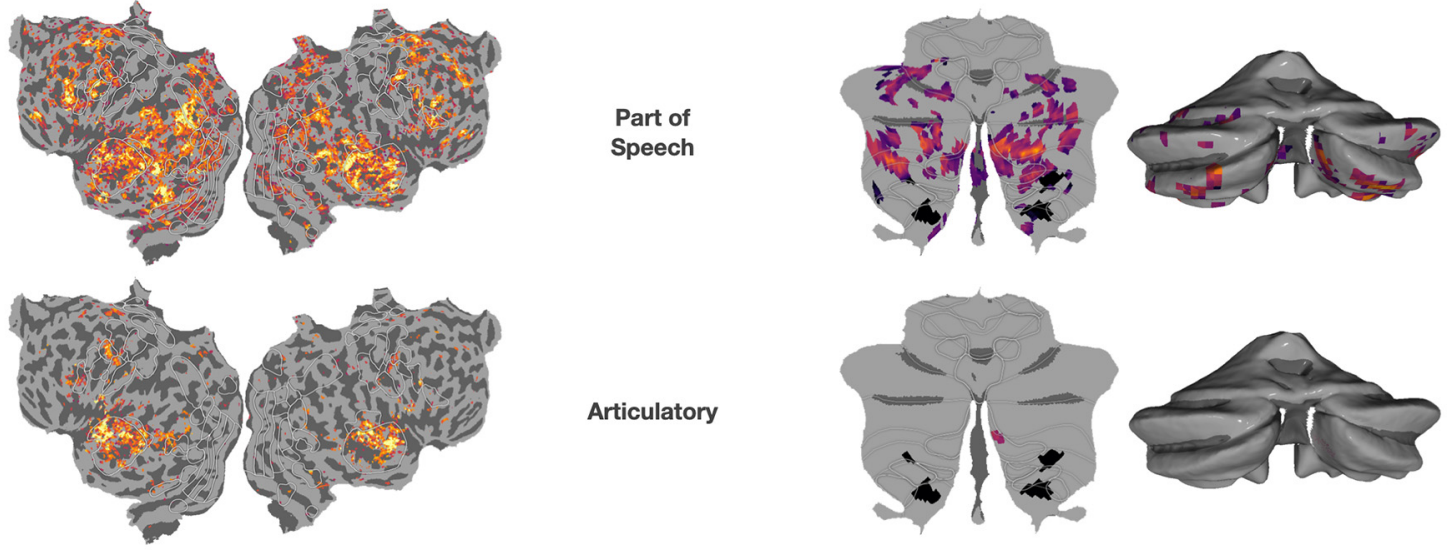

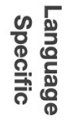

Spectral
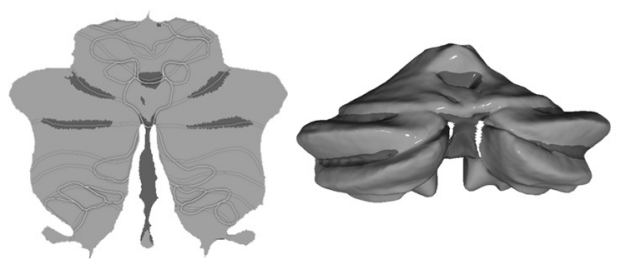

0

Prediction Performance

0.4

B

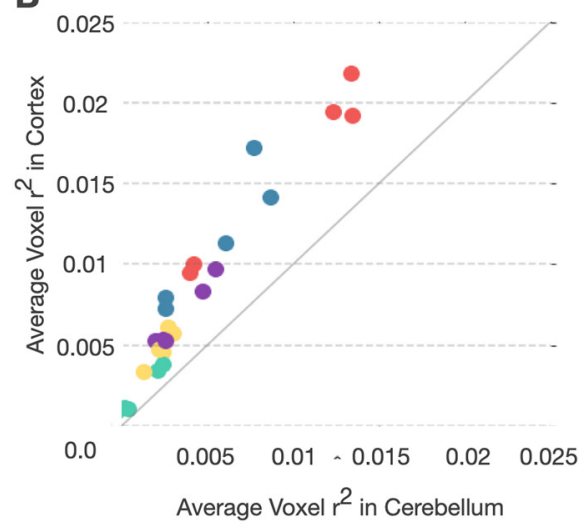

C
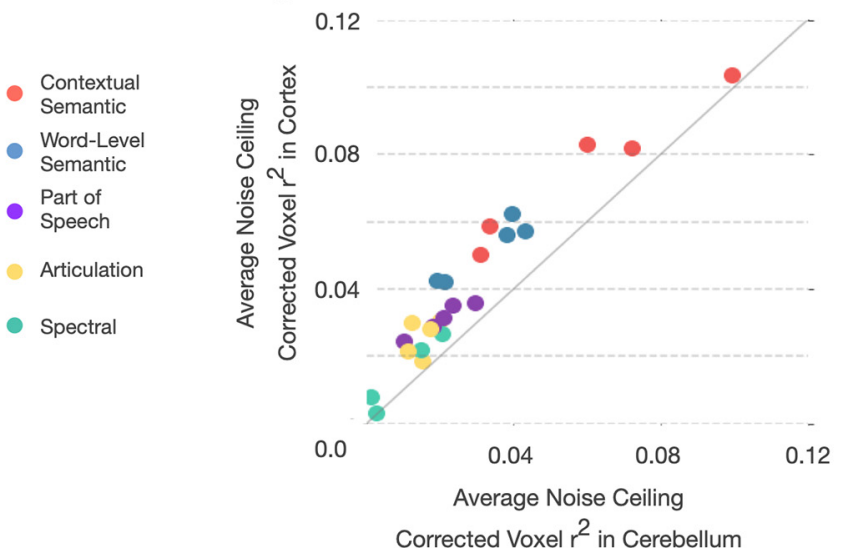

Figure 2. Prediction performance of encoding models based on five language feature spaces in cortex and cerebellum. Encoding models fit with $5.4 \mathrm{~h}$ of BOLD data were tested against a held-out story $(10 \mathrm{~min})$. $\boldsymbol{A}$, Correlation $\left(r^{2}\right)$ between predicted and actual BOLD response is plotted on flattened cortical and cerebellar surfaces for 1 subject (UT-S-02; other subjects are shown in Extended Data Fig. 2-1). Significance testing for each model in each voxel was done using a one-sided FDR-corrected permutation test with a threshold of $p<0.05$. The higher-level models 
overlapping. This could be caused by the feature spaces carrying overlapping information with each other, making it difficult to interpret the results from each feature space independently. To disentangle these representations and explore the differences between cortex and cerebellum in more detail, we next performed a variance partitioning analysis.

\section{Variance partitioning}

The previous model comparison found that many voxels in the cerebellum can be significantly predicted by multiple feature spaces. These voxels might genuinely represent information from multiple feature spaces. Indeed, the increased neuronal density of cerebellum compared with cortex (Herculano-Houzel, 2010) raises the chance that individual cerebellar voxels contain information from multiple feature spaces. However, this effect could also be a consequence of correlations, or shared information, between the feature spaces. To disentangle possible overlaps in information across the five feature spaces within each voxel, we used variance partitioning, a statistical technique for determining how much variance can be uniquely explained by each set of features (Lescroart et al., 2015; de Heer et al., 2017). This enables us to distinguish between overlapping but distinct representations and seemingly overlapping representations that actually reflect correlations between features. For example, variance partitioning would allow us to disentangle if, for example, $50 \%$ of the voxel responds to conceptual information and another $50 \%$ to auditory information, or if $100 \%$ of the voxel response is to some feature that is correlated with both auditory and conceptual information.

Apparent correlations between feature spaces can be caused by many factors, such as regions of silence and speech, which are correlated across all the feature spaces. While large datasets cannot reduce these correlations, they can enable the regression model to better account for the stimulus correlations. Our first variance partitioning analysis shows how much variance each feature space uniquely explains above all other feature spaces for each voxel, and the second shows how much overlap there is between each feature space and the context-level semantic feature space (for a correlation matrix of the feature spaces, see Extended Data Fig. 3-5).

\section{Unique variance explained}

The results in Figure $2 A$ showed negligible, localized prediction performance of low-level models in the cerebellum, suggesting that little low-level language processing was occurring there.

\footnotetext{
$\leftarrow$

have better prediction performance in both cerebellum and cortex. To confirm this, we also tested a low-level spectrotemporal modulation model, which was not substantially more predictive than the spectral model (see Extended Data Fig. 2-3). In cortex, the areas best predicted by each of the three feature categories are spatially distinct. However, in the cerebellum, the areas best predicted by each feature space are highly overlapping. $\boldsymbol{B}$, To compare across subjects, we plotted average signed $r^{2}$ across all voxels in the cerebellum and cortex for each subject and each feature space. The context-level semantic feature space has the highest predictive performance in both the cerebellum and cortex for all subjects. Performance scales roughly linearly in both cerebellum and cortex across the hierarchy of language representations, albeit with higher $r^{2}$ in cortex than cerebellum. $\boldsymbol{C}$, Because cortical and cerebellar BOLD responses might have different levels of noise, which could obscure differences in representation, we also computed noise ceiling-corrected correlations (Schoppe et al., 2016). This correction caused the average $r^{2}$ to be less biased in favor of cortex (for corrected correlation flatmaps, see Extended Data Fig. 2-2) and suggests that each feature space might be represented to a similar extent in cerebellum and cortex. However, overlapping prediction performance between different feature spaces in the cerebellum suggests that the cerebellum may not be separately representing each stage of language processing.
}

However, that result did not account for the possibility that higher-level feature spaces could also capture some low-level information. To test for this, we used variance partitioning to find the unique variance explained of each feature space to test whether the lower-level models have any unique contribution to representations in the cerebellum. This was done by first fitting a five-way union encoding model with a concatenation of all the feature spaces. Variance explained by any of the five feature spaces should be explained by this five-way union model. Then we fit five additional encoding models, each combining four of the five feature spaces. Each of these models should explain all the variance captured by the five-way union model, except for variance, which is uniquely explained by the feature space that was left out. To estimate the unique variance explained by each feature space, we then subtracted the variance explained in the four-way model, excluding that feature space from the five-way union model (for additional details, see Materials and Methods). We also used these models to estimate the size of the nonunique partition, which contains any variance that can be explained by more than one of the five feature spaces. The size of this partition was calculated by subtracting each of the unique variance partitions from the five-way union model. If a model is meaningfully represented in the cerebellum or cortex, you would expect that unique variance explained to be $>0$. For this analysis, we only considered voxels that were significantly predicted by the fiveway union model (one-sided permutation test, $q$ (FDR) $<0.05$ ).

Figure $3 A$ shows the unique variance explained by each feature space as well the nonunique partition for each voxel in the cerebellum and cortex projected onto the flattened surface for one subject (other subjects can be seen in Extended Data Fig. 3-1). The variance partition estimates were corrected for noise resulting in overfitting of the joint-model (for more details, see Materials and Methods). To see the results without correction, see Extended Data Figure 3-2. The nonunique partition is the largest partition overall, suggesting that much of the variance explained by these feature spaces cannot be specifically allocated to one feature space. It is important to note that this category includes all possible combinations of the feature spaces and does not mean that the variance is explained equally well by each of the five feature spaces. Among the unique partitions, only the context-level semantic feature space explains variance significantly $>0$ (two-sided permutation test, $q$ (FDR) $<0.05$ ). Figure $3 B$ shows the unique variance explained for each feature space averaged across voxels for all subjects (only including voxels that were significantly predicted by the union model). We compared mean partial correlations $\left(\sqrt{r^{2}}\right)$ between cerebellum and cortex for each partition using a permutation test. The result shows that all the unique partitions explain significantly less variance in the cerebellum than in cortex. When correcting for differences in signal-tonoise (Extended Data Figs. 3-3 and 3-4), the context-level semantic and word-level semantic feature spaces uniquely explain significantly more variance in the cerebellum than cortex, and the spectral feature space uniquely explains significantly less. Both of these results suggest that the cerebellum is primarily representing language at a conceptual level and that these results are not simply because of neuronal pooling within voxels or shared representations. However, the fact that the largest proportion of variance is in the nonunique partition means that this analysis alone cannot rule out the possibility for low-level language representations in the cerebellum.

\section{Pairwise partitioning}

In the first variance partitioning analysis, we found that the context-level semantic feature space explains the most unique 
A
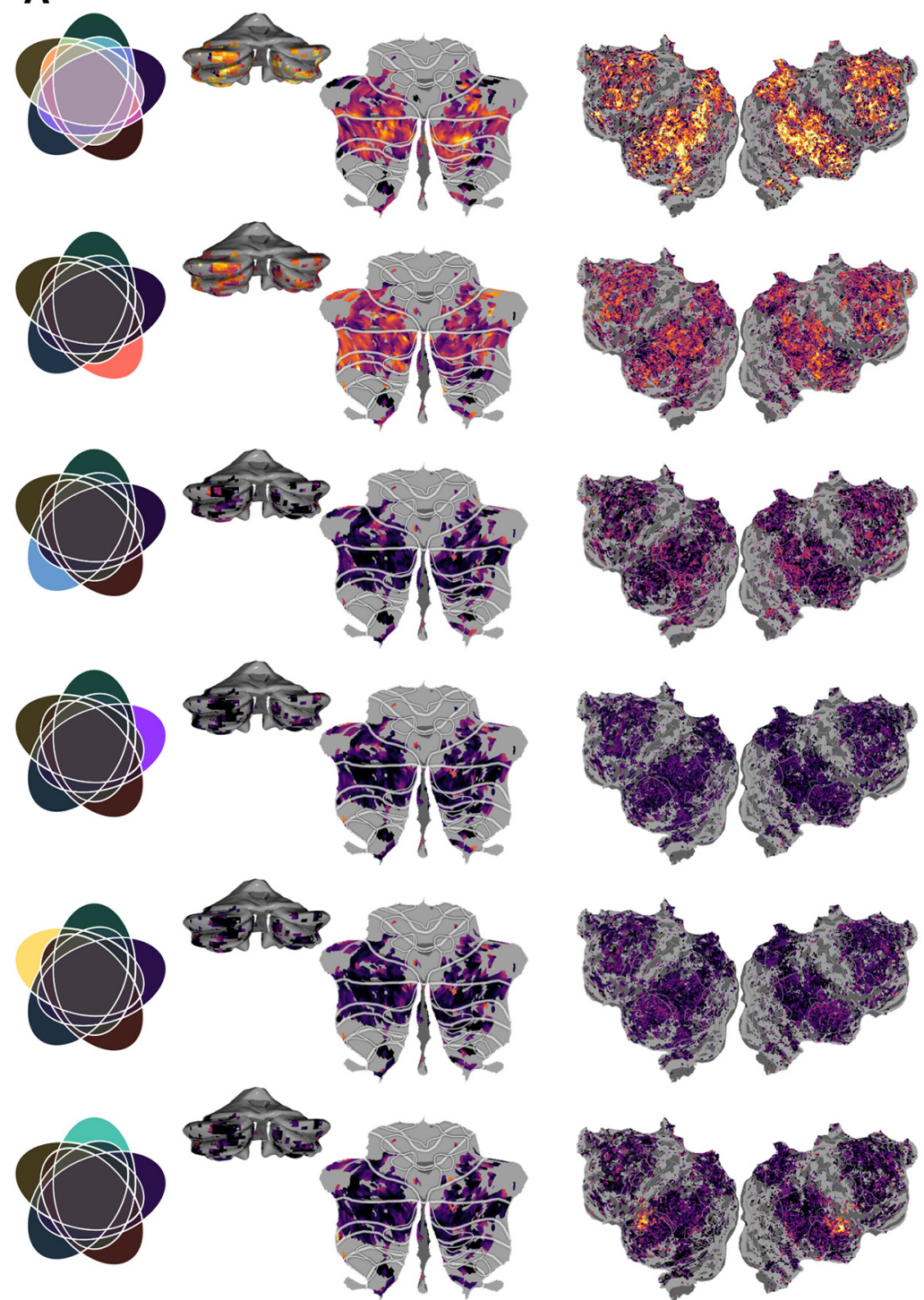

* = significantly different $(q(F D R)<0.05)$

\# = significantly greater than zero $(\mathrm{q}(\mathrm{FDR})<0.05)$

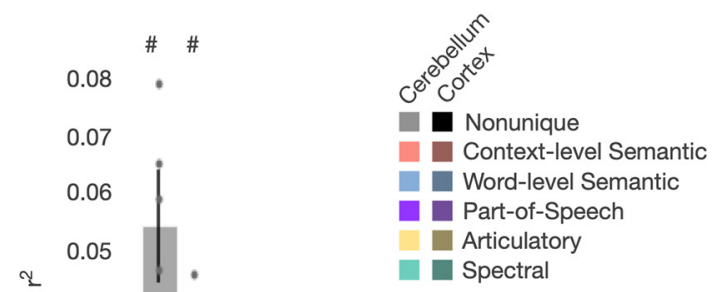

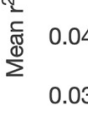

0.02

0.01

0

Not Significant
$q($ FDR $)>0.05$
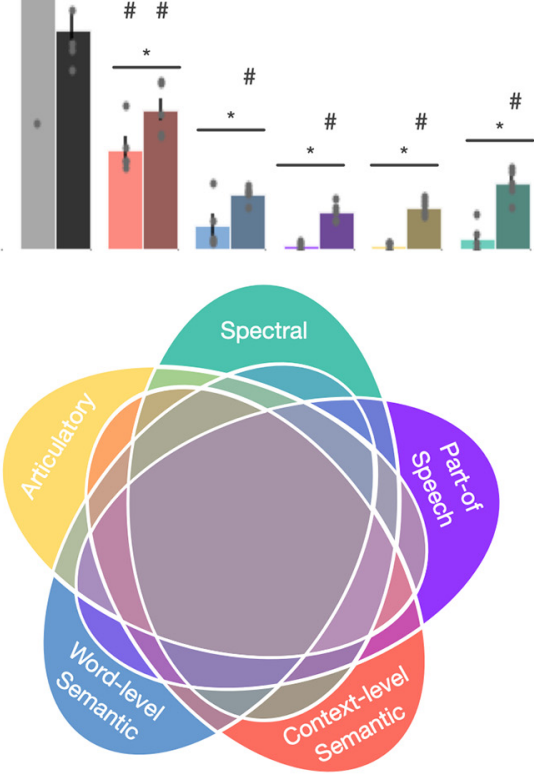

Figure 3. Unique variance explained by each feature space. To determine how much variance is uniquely explained by each feature space, six new encoding models were fit: a union model containing a concatenation of all feature spaces, and five encoding models each containing a concatenation of four of the five feature spaces (for the correlation matrix for all feature spaces, see Extended Data Fig. 3-5). The unique contribution of each feature space was then determined by subtracting the variance explained by the four-way concatenation model without that feature space from the union model. This shows how much variance can be explained by each feature space above and beyond the other four. Additionally, the amount of nonunique variance (i.e., any that can be explained by more than one feature space) was determined by subtracting the five unique variances from the union. $A$, The voxelwise partial correlation ( $\sqrt{\left(\text { partial } r^{2}\right)}$ ) for each feature space for Subject UT-S-02, projected onto the cortical and cerebellar surfaces (for similar maps for other subjects, see Extended Data Fig. 3-1). Only voxels that were significantly predicted (one-sided permutation test, $q(\mathrm{FDR})<0.05)$ by the five-way union model are displayed. For version without bias correction, see Extended Data Figure 3-2. For noise-corrected versions, see Extended Data Figures 3-3 and 3-4. B. Mean correlations for significant voxels in the cerebellum and cortex across all subjects. Errors bars are standard error of the mean. The nonunique partition contains the most variance in both cortex (darker) and cerebellum (lighter). All models explain significantly less variance in cerebellum than cortex (two-sided permutation test, $q$ $(F D R)<0.05)$. Only the context-level semantic model explains significantly $>0$ unique variance in the cerebellum. Additionally, the modality-specific feature spaces do not uniquely explain any significant variance (two-sided permutation test, $q(\mathrm{FDR})<0.05$ ), while the context-level semantic space uniquely explains the most variance. This further supports the hypothesis that the cerebellum is largely representing language at a high, conceptual level.

variance explained and that the spectral model explains significantly less variance in the cerebellum than in cortex. This suggests that the cerebellum may not be representing information at modality- and language-specific levels. However, the largest partition in both the cerebellum and cortex was the nonunique partition, which contains variance that could be explained by more than one feature space. Thus, that analysis alone cannot rule out the possibility that low-level features are represented in cerebellum. To test the hypothesis that the cerebellum is exclusively representing language at a conceptual level, we performed a second variance partitioning analysis where each feature space was separately compared with the context-level semantic feature space. We fit four union models by concatenating the contextlevel semantic features with each one of the four other feature spaces. The variance explained by each union model was then compared with models fit with each feature space individually to determine both the unique contribution of each feature space and the size of their intersection. For each pair of feature spaces, analyses were restricted to voxels that were significantly predicted by the union model. If the cerebellum was only representing information at the conceptual level, we would expect to find low unique variance explained by the modality- and language- 
specific feature spaces and a high shared intersection with the word-level semantic feature space.

The results of this pairwise variance partitioning analysis replicate previous results (de Heer et al., 2017), showing that in cortex there is a unique contribution of both the spectral and articulatory feature spaces in different cortical areas. However, this does not appear to be true in the cerebellum. Figure 4 shows the results of pairwise variance partitioning between the contextlevel semantic feature space and each of the other four feature spaces. Figure $4 A$ shows the mean partial correlation for each pair of feature spaces in both the cerebellum and cortex across voxels and subjects. The variance explained by the intersection of each pair of models is significantly less in the cerebellum than in cortex (two-sided permutation test, $q(\mathrm{FDR})<0.05$ ). This shows that the information present in the lower-level feature spaces contributes less to the explainable variance in the cerebellum and supports the hypothesis that the cerebellum is primarily representing high-level, conceptual information. Additionally, the unique contribution from the modality- and language-specific feature spaces are negligible; the spectral feature space explains significantly less variance in the cerebellum, while the articulatory feature spaces explain more variance in the cerebellum, although this partition is small in both the cerebellum and cortex. Since the intersection of these models is not zero, we cannot rule out the possibility that language- and modality-specific information is represented in cerebellum. Still, the lack of unique variance explained by these feature spaces shows that these feature spaces do not offer any more insight into cerebellar BOLD responses. When accounting for differences in signal-to-noise (Extended Data Figs. 4-3, 4-4, and 4-5), all of the unique contributions from the secondary models become significantly less in the cerebellum than in cortex. Additionally, the differences in the intersections between the cerebellum and cortex are no longer significant. However, the unique contribution from the contextlevel semantic feature space is significantly larger in all cases in the cerebellum and cortex. While the noise-ceiling corrected results are different because of the differences in BOLD signal in the cerebellum compared with cortex, the significantly larger variance explained by the context-level semantic feature space in the cerebellum still supports the hypothesis that the cerebellum is uniquely representing highly conceptual semantic information. Figure $4 B$ shows these results for one subject projected onto the corresponding cortical and cerebellar surfaces (for cortical maps for all other subjects, see Extended Data Figs. 4-1 and 4-2). Only voxels that were significantly predicted by the union model (onesided permutation test, $q(\mathrm{FDR})<0.05)$ are displayed.

Very little variance in the cerebellum is explained uniquely by any features other than the context-level semantic space. In both the cerebellum and cortex, there is a high amount of variance explained by the context-level semantic feature space and in the intersection with the word-level semantic feature space. This is not surprising, given that the context-level semantic space has the highest predictive performance of any of the feature spaces and that the word-level and context-level semantic spaces contain related semantic information. However, there is very little overlap of variance explained between the context-level semantic feature space and the three modality- and language-specific feature spaces. This demonstrates that the high performance of the conceptual feature spaces is not merely because of this feature space being correlated with low-level information. The negligible unique contribution of the modality- and language-specific features in the cerebellum further supports the hypothesis that the cerebellum is primarily representing conceptual representations.
Finally, any variance explained by the modality- and languagespecific feature spaces is not anatomically localized within cerebellum, which suggests that the cerebellum does not contain localized low-level language processing areas. The reduced representation of language-specific feature spaces in the cerebellum further suggests that the cerebellum does not participate in language processing per se, but supports cognition more generally.

\section{Semantic selectivity within the cerebellum}

Our results thus far suggest that the cerebellum is not involved with language-specific processing, as there is little or no unique variance explained in cerebellum by the part-of-speech, articulatory, or spectral feature spaces. Instead, language representations in the cerebellum appear to be dominated by conceptual semantic features. Yet all semantic representations are not alike: in cortex, earlier work revealed a patchwork tiling of areas that represent different semantic categories across much of prefrontal, parietal, and temporal cortex (Noppeney and Price, 2004; Binder et al., 2009; Huth et al., 2016). It is possible that the cerebellum represents a different range of semantic categories than cortex, and it seems likely that different categories are represented in distinct areas within the cerebellum. Following the procedure detailed by Huth et al. (2016), we used the word-level semantic feature space to analyze and interpret the model weights and thus reveal the semantic selectivity of each voxel in the cerebellum. Because of the lack of tools currently available for interpreting context-level semantic models, we chose to use the word-level model, which also explains a large proportion of response variance in the cerebellum.

To demonstrate how encoding models can be analyzed per voxel, Figure $5 \mathrm{~A}$ shows the word-level semantic regression weights projected into a three-dimensional semantic space that was previously constructed from a group of subjects using principal components analysis (Huth et al., 2012). This lowerdimensional space is purely used for visualization purposes. Here projections on the first, second, and third principal components are mapped into the red, green, and blue color channels, respectively, for each voxel and then projected onto the SUIT cerebellar surface. The color wheel shows approximately which semantic category each color on the maps represents. Figure $5 A$ shows the posterior view of one subject's (UT-S-02) cerebellum as well as the flattened cerebellar surface in SUIT space. Within the SUIT space, functional ROIs are mapped out, which include anterior foot $(\mathrm{AF})$, hand $(\mathrm{AH})$, and mouth( $\mathrm{AM})$; posterior foot $(\mathrm{PF})$, hand $(\mathrm{PH})$, and mouth $(\mathrm{PM})$; and anterior and posterior eye movement areas ( $\mathrm{AE}$ and $\mathrm{PE}$, respectively) that are active during saccades. A histogram of correlations for all voxels in Subject UT-S-02 is shown in Figure $5 B$. This histogram shows a distribution with a long tail, with the example well-predicted voxel (voxel 1685) marked in blue. Additionally, Figure $5 A$ lists the four words that the word-level semantic encoding model predicts will elicit the largest response in this example voxel, which are "voice," "silence," "shout," and "aloud." These words were found by taking the dot product of the voxel weight vector with the word-level semantic feature matrix (for details, see Materials and Methods). This voxel seems selective for concepts related to social communication and sound. Similar analysis could be performed for each voxel but would be large and difficult to interpret. However, by representing semantic weights as a color, we can better understand large-scale patterns of semantic information. For example, Crus I and Crus II seem to be selective for many different semantic categories, such as social and violence, which can be found in medial Crus I. 
A

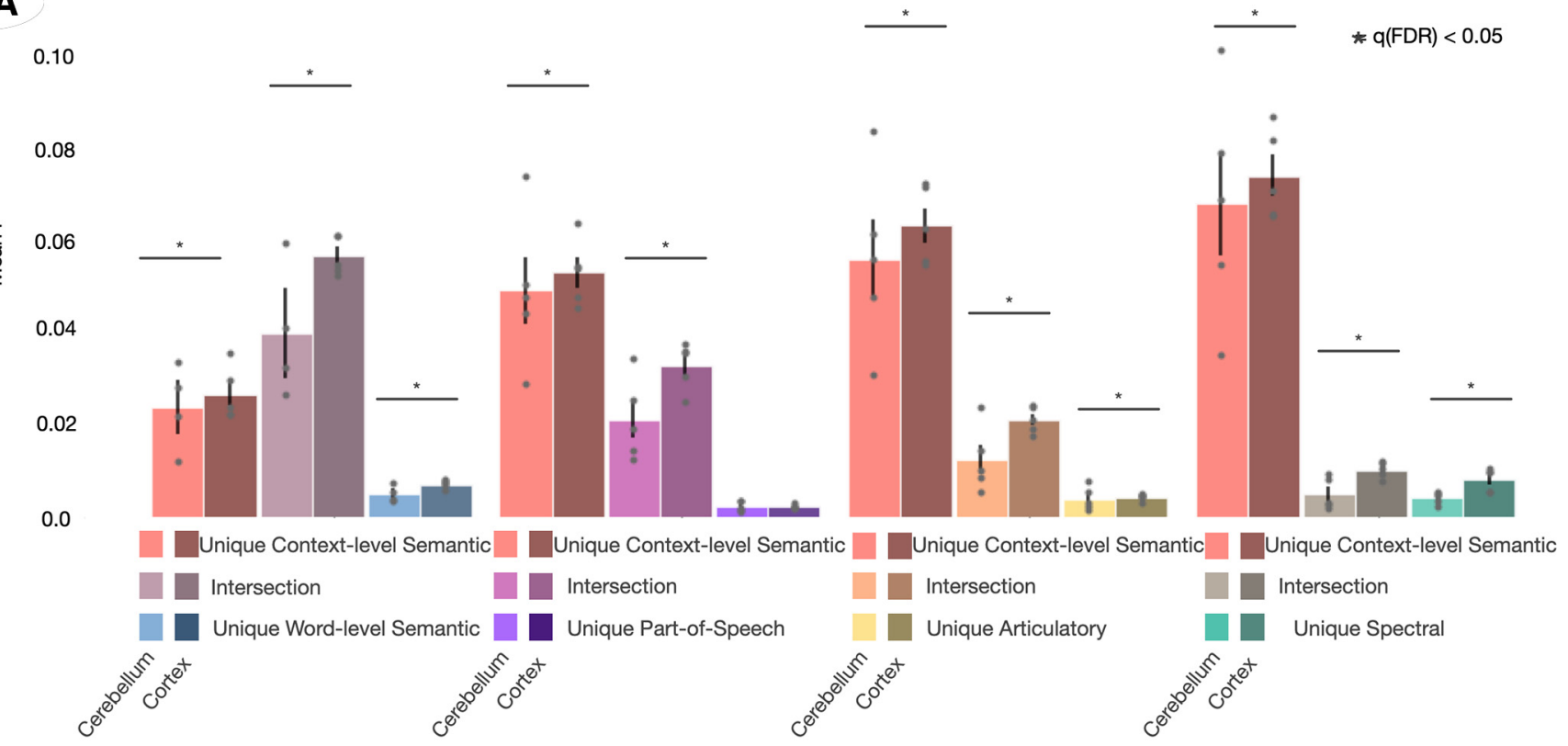

B
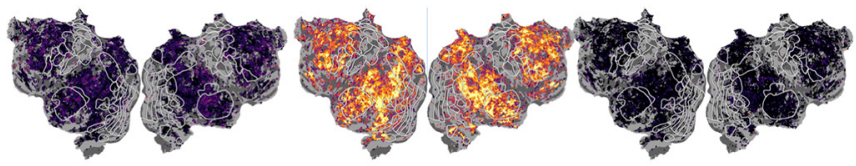

Word-Level Semantic
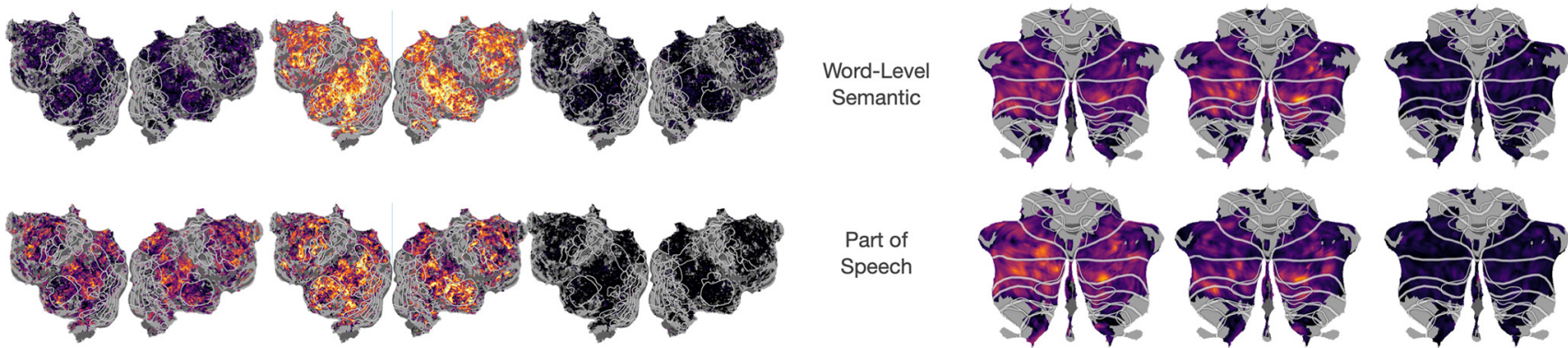

Part of

Speech
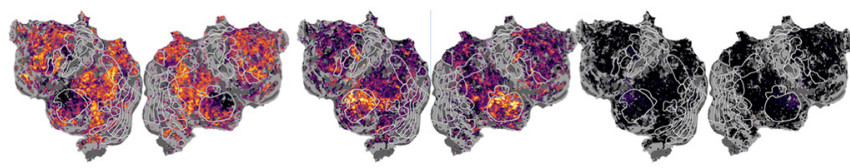

Articulatory
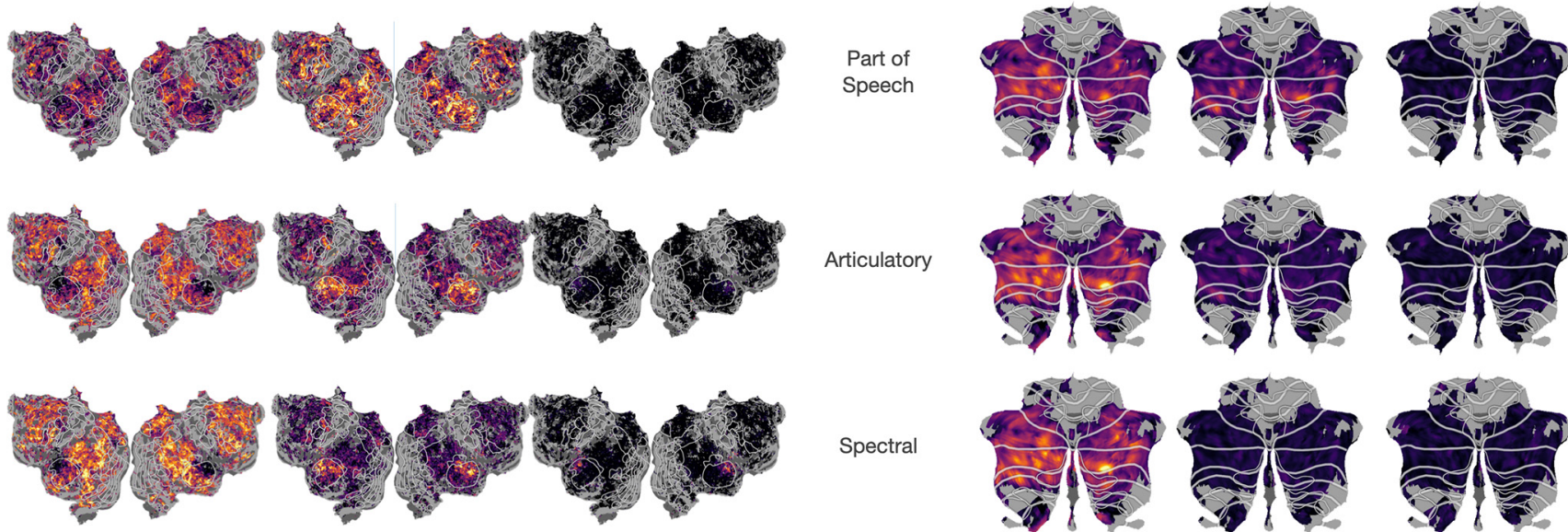

Spectral
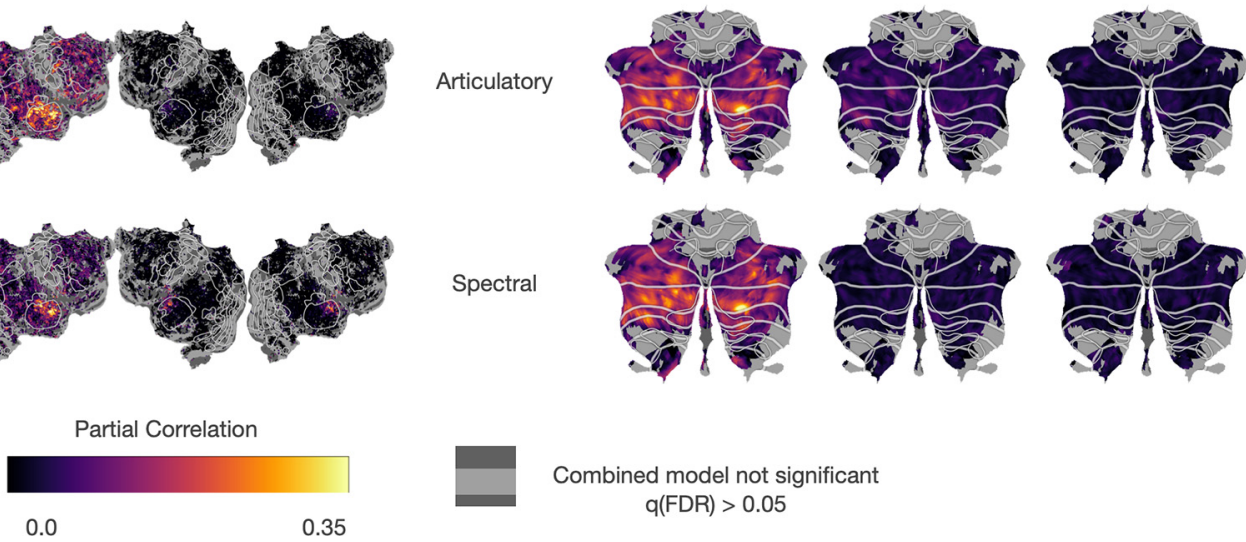

Combined model not significant $q($ FDR $)>0.05$

Figure 4. Variance partitioning between the context-level semantic feature space and each of the other feature spaces. To quantify the amount of overlap between the context-level semantic feature space and each of the four other feature spaces, three models were fit for each pair of feature spaces, including the concatenation of both feature spaces and each feature space individually. $\boldsymbol{A}$, For each pair of feature spaces, the variance uniquely explained by the context-level feature space, the variance uniquely explained by the second feature space, and the intersection between the two is compared between the cerebellum and cortex, averaged over all subjects. Error bars are the standard error of the mean. The intersection (variance that could be explained by either feature space) for every pair is smaller in the cerebellum than in the cortex (two-sided permutation test, $q(\mathrm{FDR})<0.05$ ). Additionally, the unique partition for the spectral feature space is significantly smaller in the cerebellum than in cortex. This shows that the high prediction performance of the context-level semantic feature space in cerebellum is not merely because of correlations with modality- and language-specific information. Instead, the context-level features uniquely explain a large amount of variance that the other features cannot. $B$, For each pair of models, the variance in each partition in each voxel $\left(\sqrt{\left(\text { partial } r^{2}\right)}\right)$ was projected onto cortical and cerebellar flatmaps. For other subjects, see Extended Data Figures 4-1 and 4-2. For noise-ceiling corrected versions, see Extended Data Figures 4-3, 4-4, and 4-5. Only voxels that were significantly predicted by each union model (one-sided permutation test, $q$ $(F D R)<0.05)$ are shown. There is substantially lower variance explained by the intersection between the context-level semantic feature space and the language- and modality-specific feature spaces in the cerebellum than in cortex. Additionally, the unique contributions for these feature spaces in the cerebellum are near zero and are not spatially localized. This lack of spatial localization further suggests that there is no hierarchy of language processing in the cerebellum, and these results provide strong support for the hypothesis that the cerebellum only represents high-level, conceptual features of language, rather than low-level features. 

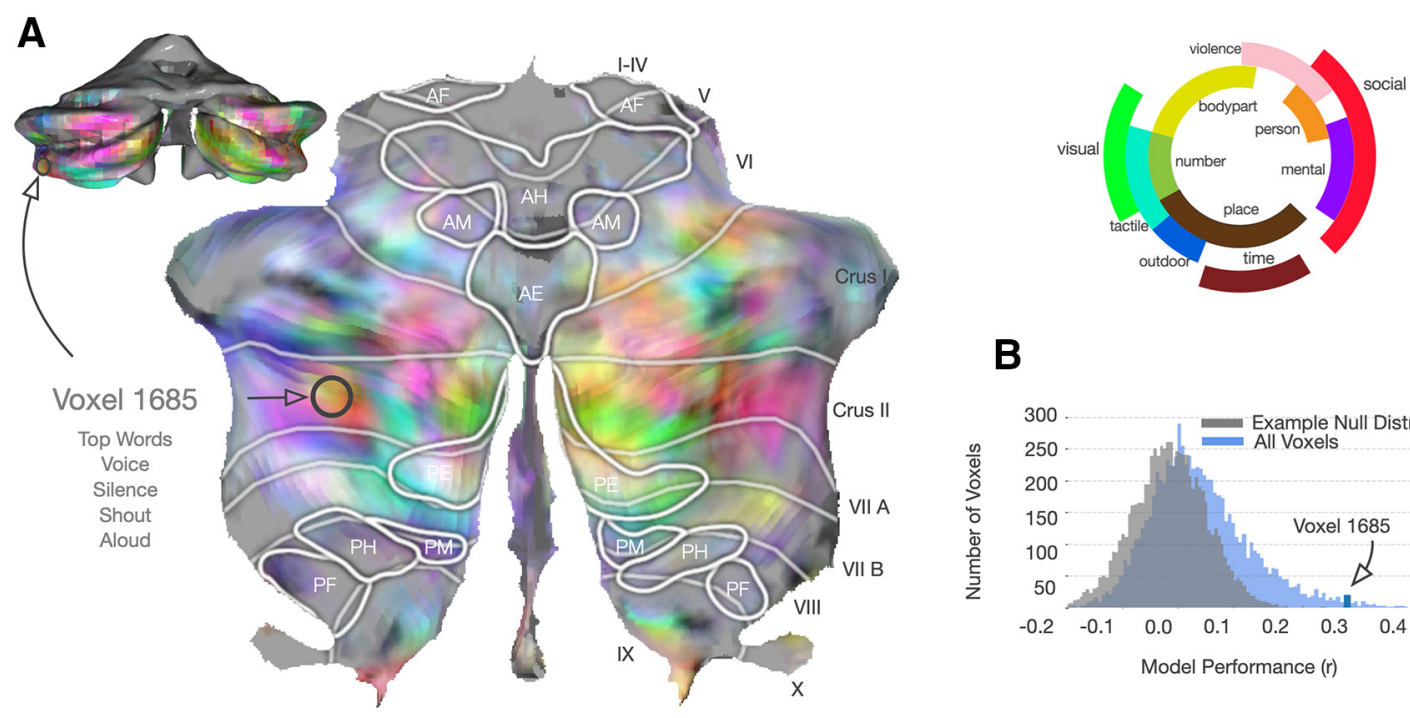

B

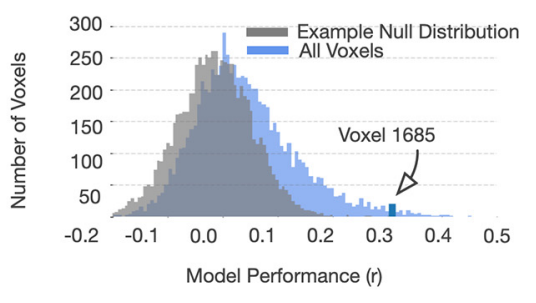

Figure 5. Word-level semantic model weight interpretation. Post hoc analysis of encoding models enables us to interpret what type of semantic information is represented in each voxel. Here we used the word-level semantic feature space to interpret one individual voxel and to broadly map semantic representations across the cerebellum. (While the context-level semantic space is more predictive, we lack tools for interpreting its representations.) In the word-level space, encoding models predict the response of each voxel to each word. We used the model to find words with the largest predicted response in one voxel (voxel 1685 in Subject UT-S-02), which were "voice," "silence," and "shout," suggesting that this voxel represents concepts related to social communication. To visualize representations across many voxels, we reduced the encoding model weights to three dimensions by projecting them onto a low-dimensional semantic space identified in a previous experiment (Huth et al., 2016), and then mapping these projections to RGB color channels. $A$, The RGB values for each voxel are projected onto the SUIT cerebellar surface for Subject UT-S-02. Different colors correspond to selectivity for different concepts in the semantic space (illustrated by the legend, right). This map suggests that the cerebellum contains representations of many different concepts. $\boldsymbol{B}$, Histogram represents the range of correlations for each voxel in this subject: blue represents the example subject; gray represents the null distribution.

\section{Comparing semantic representations between cerebellum and cortex}

The semantic map in Figure $5 \mathrm{~A}$ shows that different areas in the cerebellum represent different categories of words. Yet it is not clear from this map whether semantic representations in the cerebellum are similar to those found in cortex. To quantify the semantic categories represented in the cerebellum and cortex, we compared the fraction of voxels that represent different semantic categories using a cluster analysis. We concatenated model weights for the top $20 \%$ best predicted voxels in cerebellum and cortex from each subject, then clustered the voxels into 5 discrete categories using spherical $k$-means clustering ( 5 clusters was the elbow point of the inertia curve; see Extended Data Fig. 6-1; similar results are also obtained with different numbers of clusters). Figure $6 A, B$ shows cerebellar and cortical flatmaps with the clustered voxels colored according to their assigned cluster in one subject (similar maps for other subjects can be found in Extended Data Fig. 6-2). The label for each cluster was determined qualitatively from the most similar words to each cluster centroid (Fig. 6C lists the clusters, their top words, and their assigned label; secondary cluster labels generated by external observers also available in Extended Data Fig. 6-3).

Voxels belonging to every semantic cluster were found in both the cerebellum and cortex. Figure $6 C$ shows the percentage of cerebellar voxels in each cluster compared with the percentage of cortical voxels in each cluster, averaged across subjects. The category with the highest percentage of voxels in the cerebellum is the "people" category, and the category with the lowest percentage is the "place" category.

Because voxels in all clusters are found in both the cerebellum and cortex, it is possible that the cerebellum is receiving input from all areas of cortex. If this were true, we would expect to find an equal percentage of well-predicted cerebellar voxels in each cluster as there are in cortex. However, all clusters had significantly different percentages of voxels in the cerebellum compared with cortex (two-sided permutation test, $q(\mathrm{FDR})<0.05)$. The "social" and "people" clusters have a higher percentage of voxels in the cerebellum than in cortex, and the "mental," "concrete," and "place" clusters have a lower percentage of voxels in cerebellum than in cortex. This suggests that there is not a one-to-one mapping from cortex to the cerebellum and that the cerebellum is more responsive to social semantic information.

\section{Discussion}

This study examined how language is represented in the human cerebellum. Using voxelwise encoding models trained within each subject using large amounts of fMRI data, we found that high-level language feature spaces (context-level and word-level semantics) were better able to predict cerebellar BOLD responses than low-level language feature spaces, such as part-of-speech and articulations. Additionally, the low-level feature spaces do not uniquely predict any voxel in the cerebellum above the context-level semantic model, which is not true in the cortex. Last, using the model weights from the word-level semantic model, we found that there is an overrepresentation of social and people semantic categories in the cerebellum compared with cortex. These results suggest that (1) the cerebellum is representing language at a conceptual level, and not at modality- or languagespecific levels; (2) there is not a homologous area to auditory cortex in the cerebellum; and (3) the cerebellum is more responsive to social semantic components of language than cortex. As has been seen previously (King et al., 2019), there does not appear to be any functional relevance to lobule boundaries as we do not observe any pattern of language processing that corresponds to the lobule boundaries.

One complication in interpreting the results of this study is because of the use of BOLD fMRI, in particular in relation to the 
A

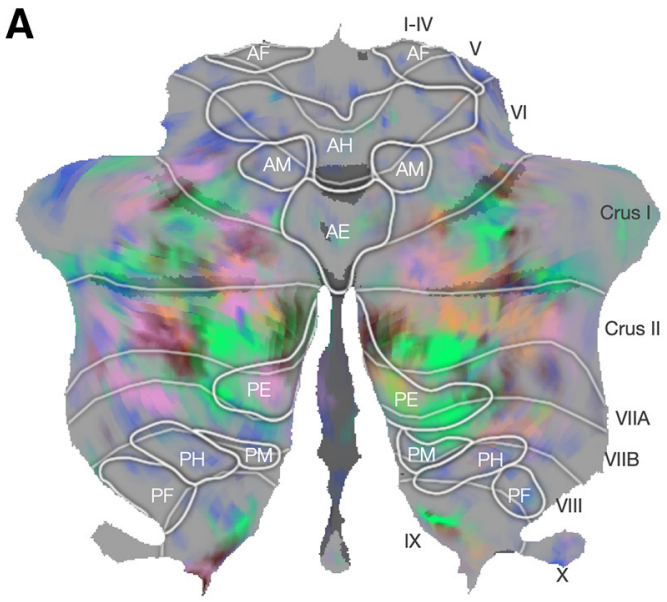

C

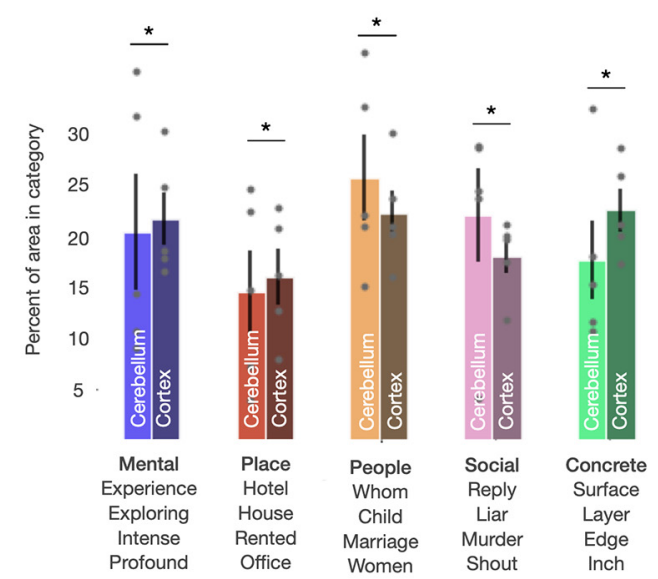

B

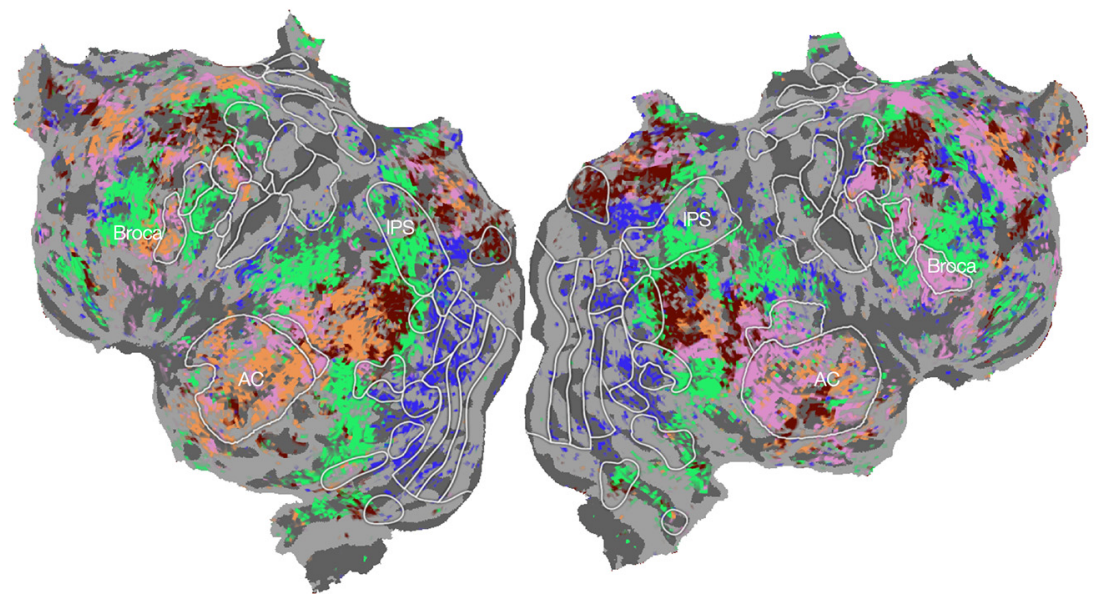

Figure 6. Differences in semantic representations between cerebellum and cortex. To check for differences in semantic representations between the cerebellum and cortex, word-level encoding model weights from both cerebellum and cortex in all subjects were concatenated, including only the top $20 \%$ best-predicted voxels. This matrix was then clustered using spherical $k$-means into 5 clusters, which fell at the inflection point in the inertia graph (Extended Data Fig. 6-1). For visualization, the centroid for each cluster was transformed into the same RGB space used in Figure 5, and each voxel in that cluster was assigned that color. $\boldsymbol{A}, \boldsymbol{B}$, The cluster distribution for Subject UT-S-02 across the cerebellum $(\boldsymbol{A})$ and cortex (B) (for other subjects, see Extended Data Fig. 6-2). Voxels falling into each cluster are found in both the cerebellum and cortex in every subject. $\boldsymbol{C}$, To test for differences in representation between cortex and cerebellum, the percentage of cortical and cerebellar voxels in each cluster were compared across all subjects. Each cluster was named qualitatively according to the most similar words to the cluster centroid. The four most similar words to each cluster centroid are listed below the label name. The error bars are standard error of the mean. Significantly more voxels in the cerebellum were highly responsive to social categories (two-sided permutation test, $q($ FDR $)<0.05)$ (i.e., the "social" and "people" clusters) than in cortex. Conversely, significantly fewer voxels in the cerebellum were responsive to the "mental," "concrete," or "place" clusters than in the cortex. This shows that the cerebellum is largely representing the same semantic categories as cortex, but that there is a slight bias toward social categories.

cerebellum. The cerebellum has a significantly different metabolic demand than cortex (Vaishnavi et al., 2010) because of its cellular architecture. This changes the demand for oxygenated blood and thus the BOLD signal. It has previously been demonstrated that only activity in granule cells and mossy fibers affects the BOLD signal (Mathiesen et al., 2000; Caesar et al., 2003) in the cerebellum, but not activity in the Purkinje cells, which are the sole output from the cerebellum to the cortex. This implies that our models do not include representations of what the cerebellum is outputting back to the cortex and thus may not directly address the computation the cerebellum is performing. However, the input to the cerebellum (granule cells and mossy fibers) is still an important half of the equation, and this work furthers our understanding of what kinds of representations are being sent to the cerebellum.

One point of contention with our methodology is using a natural stimulus. While natural stimuli can make interpretation of the results more difficult, it is a much richer stimulus set for analysis. Additionally, it is less biased than other experimental methods that preselect a small number of categories or stimuli. And while we have few subjects, we have collected a large amount of data per subject. While large datasets cannot reduce these correlations within the stimulus, they can enable the regression model to better account for the stimulus correlations. Additionally, by using a prediction methodology, we are able to compute the variance explained by each feature space, which allows us to quantify how well each model does at prediction which few other methods allow for.

Many theories exist for how the cerebellum represents cognitive information based on the uniformity of its cellular architecture. This architecture is believed to suggest that the cerebellum is performing a similar function throughout the structure. Additionally, the cerebellum has long been considered a major region in motor response and motor learning (Manto et al., 2012). Yet since the 1980s, the cerebellum has been known to reliably respond during cognitive tasks (Leiner et al., 1986), such as language processing (Petersen et al., 1988), and that lesions to the posterior lobe of the cerebellum result in language deficits (Schmahmann and Sherman, 1998). The fact that both fine motor control processing and cognitive processing elicit strong 
responses from the same architecture has long been considered a contradiction. In an effort to reconcile the cerebellum as both a cognitive area and a motor area, previous reports have speculated that the cerebellum is involved in some low-level component of cognitive tasks, such as low-level auditory processing (Petacchi et al., 2005) or motor planning in speech (Jürgens, 2002).

Surprisingly, our results show that low-level spectral and articulatory feature spaces do not uniquely predict any area of the cerebellum better than high-level feature spaces. However, spectral and articulatory models best predict areas around auditory cortex and the STG, as has been reported by others using fMRI with speech (de Heer et al., 2017) as well as more diverse natural sounds (Moerel et al., 2012; Santoro et al., 2014). This shows that these feature spaces can successfully capture auditory information that is represented in cortex, even using such a slow imaging technique as BOLD fMRI. Yet this information does not appear to be present in the cerebellum. The cerebellum is very likely involved with motor components of speech production (Ackermann et al., 2007). However, we found no evidence of receptive articulatory representations in the cerebellum, suggesting it must be involved in more than just motor components of speech perception (Liberman and Mattingly, 1985). Our results imply that the cerebellum lacks any form of localized low-level auditory processing area for speech sounds and that the role of the cerebellum in language processing is at a higher level than previously thought.

An important future step is to clarify the relationship between language representations in the cerebellum and existing theories of cerebellar function. The universal cerebellar transform theory is the predominant theory of cerebellar computation (Diedrichsen et al., 2019), positing that the cerebellum performs a single computation across all tasks, both cognitive and motor. A commonly proposed computation is prediction error (Kawato and Gomi, 1992; Mariën and Manto, 2018). One way to look at whether the cerebellum is involved in prediction is through surprisal, which is a measure of the probability of a word occurring in a sentence given the previous word. Thus, a word with a high surprisal is likely to have a high prediction error. Since the context-level semantic model is using a neural network-based language model, it inherently captures some elements of surprisal (Berger et al., 1996). However, the contextlevel semantic model best predicts both the cerebellum and cortex, which suggests that the cerebellum is not uniquely computing surprisal, as both cortical and cerebellar BOLD signals are modulated by surprisal.

In language processing, many processes are specific to auditory communication, such as the spectral and articulatory features spaces. However, the higher-order semantic features seem to be more broadly used by the default mode network. Given that the cerebellum does not appear to be involved in the lowerlevel language processing, our results support the hypothesis that the cerebellum is not participating in language processing per se, and is instead only involved in cognitive processing. However, given that this current work only looks at a language stimulus, we cannot rule out the possibility that these results are driven from the linguistic nature of the stimulus. This theory, that the cerebellum is cognitive, and not linguistic, could explain many of the language deficits seen in patients with CCAS and autism. Both of these disorders are associated with cerebellar damage or morphologic changes, and both often see deficits in language processing (Stoodley and Schmahmann, 2009). However, the deficits are not specifically related to speech production or the ability to interpret sound into phonemes and words, which are low-level language-specific processes. Rather, the language deficits in CCAS and autism more often present as conceptual deficits, with a loss of understanding of fine-tuned semantic specificity and social dynamics (Kelley et al., 2006), such as understanding sarcasm and nonexplicit language. Much like the cerebellum being involved in the fine-tuning of motor commands over a continuous threedimensional space, it is possible that the cerebellum is similarly involved in the fine-tuning of a conceptual cognitive space.

\section{References}

Ackermann H, Mathiak K, Riecker A (2007) The contribution of the cerebellum to speech production and speech perception: clinical and functional imaging data. Cerebellum 6:202-213.

Akbik A, Bergmann T, Blythe D, Rasul K, Schweter S, Vollgraf R (2019) FLAIR: An easy-to-use framework for state-of-the-art NLP. In: Proceedings of the 2019 Conference of the North American Chapter of the Association for Computational Linguistics (Demonstrations), pp 5459.

Allen G, Buxton RB, Wong EC, Courchesne E (1997) Attentional activation of the cerebellum independent of motor involvement. Science 275:1940-1943.

Berger AL, Della Pietra VJ, Della Pietra SA (1996) A maximum entropy approach to natural language processing. Comput Linguist 22:39-68.

Binder JR, Frost JA, Hammeke TA, Cox RW, Rao SM, Prieto T (1997) Human brain language areas identified by functional magnetic resonance imaging. J Neurosci 17:353-362.

Binder JR, Desai RH, Graves WW, Conant LL (2009) Where is the semantic system? A critical review and meta-analysis of 120 functional neuroimaging studies. Cereb Cortex 19:2767-2796.

Boersma P, Weenink D (2021) Praat: doing phonetics by computer [Computer program]. Version 6.2. Available at http://www.praat.org/.

Booth JR, Wood L, Lu D, Houk JC, Bitan T (2007) The role of the basal ganglia and cerebellum in language processing. Brain Res 1133:136-144.

Brissenden JA, Tobyne SM, Osher DE, Levin EJ, Halko MA, Somers DC (2018) Topographic cortico-cerebellar networks revealed by visual attention and working memory. Curr Biol 28:3364-3372.e5.

Buckner RL, Krienen FM, Castellanos A, Diaz JC, Yeo BT (2011) The organization of the human cerebellum estimated by intrinsic functional connectivity. J Neurophysiol 106:2322-2345.

Caesar K, Gold L, Lauritzen M (2003) Context sensitivity of activity-dependent increases in cerebral blood flow. Proc Natl Acad Sci USA 100:4239-4244.

Callan DE, Tsytsarev V, Hanakawa T, Callan AM, Katsuhara M, Fukuyama H, Turner R (2006) Song and speech: brain regions involved with perception and covert production. Neuroimage 31:1327-1342.

Chang S-E, Horwitz B, Ostuni J, Reynolds R, Ludlow CL (2011) Evidence of left inferior frontal-premotor structural and functional connectivity deficits in adults who stutter. Cereb. Cortex 21:2507-2518.

Cheung C, Hamilton LS, Johnson K, Chang EF (2016) The auditory representation of speech sounds in human motor cortex. Elife 5:e17181.

Cook M, Murdoch B, Cahill L, Whelan B (2004) Higher-level language deficits resulting from left primary cerebellar lesions. Aphasiology 18:771-784.

Dale AM, Fischl B, Sereno MI (1999) Cortical surface-based analysis. I. segmentation and surface reconstruction. Neuroimage 9:179-194.

de Heer WA, Huth AG, Griffiths TL, Gallant JL, Theunissen FE (2017) The hierarchical cortical organization of human speech processing. J Neurosci 37:6539-6557.

Deniz F, Nunez-Elizalde AO, Huth AG, Gallant JL (2019) The representation of semantic information across human cerebral cortex during listening versus reading is invariant to stimulus modality. J Neurosci 39:7722-7736.

Diedrichsen J (2006) A spatially unbiased atlas template of the human cerebellum. Neuroimage 33:127-38.

Diedrichsen J, King M, Hernandez-Castillo C, Sereno M, Ivry RB (2019) Universal transform or multiple functionality? Understanding the contribution of the human cerebellum across task domains. Neuron 102:918-928.

Downing PE, Jiang Y, Shuman M, Kanwisher (2001) NA cortical area selective for visual processing of the human body. Science 293:2470-2473.

Dronkers NF, Wilkins DP, Van Valin RD Jr, Redfern BB, Jaeger JJ (2004) Lesion analysis of the brain areas involved in language comprehension. Cognition 92:145-177.

Epstein R, Kanwisher NA (1998) cortical representation of the local visual environment. Nature 392:598-601.

Fedorenko E, Behr MK, Kanwisher N (2011) Functional specificity for highlevel linguistic processing in the human brain. Proc Natl Acad Sci USA 108:16428-16433. 
Fedorenko E, Duncan J, Kanwisher N (2013) Broad domain generality in focal regions of frontal and parietal cortex. Proc Natl Acad Sci USA 110:16616-16621.

Fiez JA, Petersen SE, Cheney MK, Raichle ME (1992) Impaired non-motor learning and error detection associated with cerebellar damage: a single case study. Brain 115:155-178.

Firth J (1957) A synopsis of linguistic theory, 1930-55. In Studies in Linguistic Analysis. Special Volume of the Philological Society, pp 1-31. Oxford: Blackwell.

Frank B, Schoch B, Hein-Kropp C, Hövel M, Gizewski ER, Karnath HO, Timmann D (2008) Aphasia, neglect and extinction are no prominent clinical signs in children and adolescents with acute surgical cerebellar lesions. Exp Brain Res 184:511-519.

Gao JS, Huth AG, Lescroart MD, Gallant JL (2015) Pycortex: an interactive surface visualizer for fMRI. Front Neuroinform 9:23.

Herculano-Houzel S (2010) Coordinated scaling of cortical and cerebellar numbers of neurons. Front Neuroanat 4:12

Hickok G, Poeppel D (2007) The cortical organization of speech processing. Nat Rev Neurosci 8:393-402.

Huth AG, Nishimoto S, Vu AT, Gallant JL (2012) A continuous semantic space describes the representation of thousands of object and action categories across the human brain. Neuron 76:1210-1224.

Huth AG, de Heer WA, Griffiths TL, Theunissen FE, Gallant JL (2016) Natural speech reveals the semantic maps that tile human cerebral cortex. Nature 532:453-458.

Jain S, Huth AG (2018) Incorporating context into language encoding models for fMRI. Adv Neural Information Process Syst 2018:6628-6637.

Jürgens U (2002) Neural pathways underlying vocal control. Neurosci Biobehav Rev 26:235-258.

Justus T (2004) The cerebellum and English grammatical morphology: evidence from production, comprehension, and grammaticality judgments. J Cogn Neurosci 16:1115-1130.

Kanwisher N, McDermott J, Chun MM (1997) The fusiform face area: a module in human extrastriate cortex specialized for face perception. J Neurosci 17:4302-4311.

Kawato M, Gomi H (1992) A computational model of four regions of the cerebellum based on feedback-error learning. Biol Cybern 68:95-103.

Kelley E, Paul JJ, Fein D, Naigles LR (2006) Residual language deficits in optimal outcome children with a history of autism. J Autism Dev Disord 36:807-828.

King M, Hernandez-Castillo CR, Poldrack RA, Ivry RB, Diedrichsen J (2019) Functional boundaries in the human cerebellum revealed by a multi-domain task battery. Nat Neurosci 22:1371-1378.

Kunsch HR (1989) The jackknife and the bootstrap for general stationary observations. Ann Stat 17:1217-1241.

LeBel A, Wagner L, Jain S, Adhikari-Desai A, Gupta B, Morgenthal A, Tang J, Xu L, Huth AG (2021) An fMRI dataset during a passive natural language listening task. OpenNeuro. doi:10.18112/openneuro.ds003020. v1.0.2.

Leiner HC, Leiner AL, Dow RS (1986) Does the cerebellum contribute to mental skills? Behav Neurosci 100:443-454.

Lescroart MD, Stansbury DE, Gallant JL (2015) Fourier power, subjective distance, and object categories all provide plausible models of BOLD responses in scene-selective visual areas. Front Comput Neurosci 9:135.

Levelt WJ (1993) Speaking: from intention to articulation. Cambridge, MA: Massachusetts Institute of Technology.

Liberman AM, Mattingly IG (1985) The motor theory of speech perception revised. Cognition 21:1-36.

Lin Y, Tan YC, Frank R (2019) Open sesame: getting inside BERT's linguistic knowledge. arXiv:1906.01698.

Liu L, Ioannides AA, Streit M (1999) Single trial analysis of neurophysiological correlates of the recognition of complex objects and facial expressions of emotion. Brain Topogr 11:291-303.

Manto M, Bower JM, Conforto AB, Delgado-García JM, da Guarda SN, Gerwig M, Habas C, Hagura N, Ivry RB, Mariën P, Molinari M, Naito E, Nowak DA, Ben Taib NO, Pelisson D, Tesche CD, Tilikete C, Timmann D (2012)
Consensus paper: roles of the cerebellum in motor control-the diversity of ideas on cerebellar involvement in movement. Cerebellum 11:457-487.

Marek S, Siegel JS, Gordon EM, Raut RV, Gratton C, Newbold DJ, Ortega M, Laumann TO, Adeyemo B, Miller DB, Zheng A, Lopez KC, Berg JJ, Coalson RS, Nguyen AL, Dierker D, Van AN, Hoyt CR, McDermott KB, Norris SA, et al. (2018) Spatial and temporal organization of the individual human cerebellum. Neuron 100:977-993.e7.

Mariën P, Manto M (2018) Cerebellum as a master-piece for linguistic predictability. Cerebellum 17:101-103.

Mathiesen C, Caesar K, Lauritzen M (2000) Temporal coupling between neuronal activity and blood flow in rat cerebellar cortex as indicated by field potential analysis. J Physiol 523:235-246.

Moerel M, De Martino F, Formisano E (2012) Processing of natural sounds in human auditory cortex: tonotopy, spectral tuning, and relation to voice sensitivity. J Neurosci 32:14205-14216.

Murdoch BE (2010) The cerebellum and language: historical perspective and review. Cortex 46:858-868.

Noppeney U, Price CJ (2004) Retrieval of abstract semantics. Neuroimage 22:164-170.

Norman-Haignere SV, McDermott JH (2018) Neural responses to natural and model-matched stimuli reveal distinct computations in primary and nonprimary auditory cortex. PLoS Biol 16:e2005127.

Petacchi A, Laird AR, Fox PT, Bower JM (2005) Cerebellum and auditory function: an ALE meta-analysis of functional neuroimaging studies. Hum Brain Mapp 25:118-128.

Petersen SE, Fox PT, Posner MI, Mintun M, Raichle ME (1988) Positron emission tomographic studies of the cortical anatomy of single-word processing. Nature 331:585-589.

Poeppel D, Emmorey K, Hickok G, Pylkkänen L (2012) Towards a new neurobiology of language. J Neurosci 32:14125-14131.

Radford A, Narasimhan K, Salimans T, Sutskever I (2018) Improving language understanding by generative pre-training. OpenAI Blog. Available at https://openai.com/blog/language-unsupervised/.

Radford A, Wu J, Child R, Luan D, Amodei D, Sutskever I (2019) Language models are unsupervised multitask learners. OpenAI Blog 1:9.

Santoro R, Moerel M, De Martino F, Goebel R, Ugurbil K, Yacoub E, Formisano E (2014) Encoding of natural sounds at multiple spectral and temporal resolutions in the human auditory cortex. PLoS Comput Biol 10:e1003412.

Schmahmann JD, Sherman JC (1998) The cerebellar cognitive affective syndrome. Brain 121:561-579.

Schoppe O, Harper NS, Willmore BD, King AJ, Schnupp JW (2016) Measuring the performance of neural models. Front Comput Neurosci 10:10.

Silveri MC, Leggio MG, Molinari M (1994) The cerebellum contributes to linguistic production: a case of agrammatic speech following a right cerebellar lesion. Neurology 44:2047-2050.

Silveri MC, Misciagna S (2000) Language, memory, and the cerebellum. J Neurolinguistics 13:129-143.

Snider RS, Stowell A (1944) Receiving areas of the tactile, auditory, and visual systems in the cerebellum. J Neurophysiol 7:331-357.

Stoodley CJ, Schmahmann JD (2009) The cerebellum and language: evidence from patients with cerebellar degeneration. Brain Lang 110:149-153.

Tenney I, Xia P, Chen B, Wang A, Poliak A, McCoy RT, Kim N, Van Durme B, Bowman SR, Das D, Pavlick E (2019) What do you learn from context? Probing for sentence structure in contextualized word representations. arXiv.

Toneva M, Wehbe L (2019) Interpreting and improving natural-language processing (in machines) with natural language-processing (in the brain). Advances in Neural Information Processing Systems, pp 14954-14964.

Vaishnavi SN, Vlassenko AG, Rundle MM, Snyder AZ, Mintun MA, Raichle ME (2010) Regional aerobic glycolysis in the human brain. Proc Natl Acad Sci USA 107:17757-17762.

Woolrich MW, Jbabdi S, Brian Patenaude B, Chappell M, Makni S, Behrens T, Beckmann C, Jenkinson M, Smith SM (2009) Bayesian analysis of neuroimaging data in FSL. Neuroimage 45:S173-86.

Yuan MLJ (2008) Speaker identification on the SCOTUS corpus. In: Proceedings of Acoustics, pp 5687-5690 